\title{
Meson effective mass in the isospin medium in hard-wall AdS/QCD model
}

\author{
Shahin Mamedov 1,2,3,a \\ ${ }^{1}$ Department of Physics, Gazi University, Teknikokullar, 06100 Ankara, Turkey \\ 2 Institute for Physical Problems, Baku State University, Z.Khalilov 23, 1148 Baku, Azerbaijan \\ ${ }^{3}$ Institute of Physics, Azerbaijan National Academy of Sciences, H.Javid Ave. 33, 1143 Baku, Azerbaijan
}

Received: 4 August 2015 / Accepted: 25 January 2016 / Published online: 17 February 2016

(C) The Author(s) 2016. This article is published with open access at Springerlink.com

\begin{abstract}
We study a mass splitting of the light vector, axial-vector, and pseudoscalar mesons in the isospin medium in the framework of the hard-wall model. We write an effective mass definition for the interacting gauge fields and scalar field introduced in gauge field theory in the bulk of AdS space-time. Relying on holographic duality we obtain a formula for the effective mass of a boundary meson in terms of derivative operator over the extra bulk coordinate. The effective mass found in this way coincides with the one obtained from finding of poles of the two-point correlation function. In order to avoid introducing distinguished infrared boundaries in the quantization formula for the different mesons from the same isotriplet we introduce extra action terms at this boundary, which reduces distinguished values of this boundary to the same value. Profile function solutions and effective mass expressions were found for the in-medium $\rho, a_{1}$, and $\pi$ mesons.
\end{abstract}

\section{Introduction}

The isospin medium is the simplification of the dense nuclear medium, where the net baryon charge of the medium is taken zero while its isospin chemical potential remains non-zero. Such a simplified model in QCD was introduced in [1]. The AdS/CFT correspondence conjecture developed in [2-6] further was applied to QCD problems and AdS/QCD models were constructed for the mesons [7-14]. Nucleons were included into AdS/QCD models in $[15,16]$. The AdS/QCD idea was extended to the finite temperature case in [17-26] and a great number of works were devoted to a holographic description of the quark-gluon plasma and dense nuclear matter [27-29]. In the framework of holographic QCD the studies in the dense nucleon and isospin mediums turned out

a e-mail: sh.mamedov62@gmail.com to be as effective in a top-down approach [30-33] as in a bottom-up one [34-39].

In holographic QCD the two phases of nuclear matter - the confining and deconfining ones - in the dual gravity theory side are described by the different metrics. In the bottom-up approach, when the quark matter is absent, the deconfining phase at the gravity side is described by the Schwarzschild AdS black hole (SAdS BH) metric [17]. The confined phase of the nuclear matter in the low temperature limit in the dual gravity theory is described by the thermal AdS space (tAdS) [40,41]. In [42] it was shown on the background geometry of the dual gravity for the confinement phase containing the quark matter fields as well. This metric is named the thermal charged AdS space (tcAdS) metric, and it can be obtained from the Reissner-Nordstrom black hole (RNAdS BH) metric by taking a zero of the mass of black hole and cutting of the fifth dimension at the infrared (IR) boundary. The deconfinement phase in dual gravity is described by the RNAdS $\mathrm{BH}$ metric. There occurs a Hawking-Page transition between these geometries as the confinement-deconfinement phase transition takes place in the dual field theory [43-46].

One of the simplifications in nuclear matter studies using holography is in considering a zero temperature limit. Another one is taking the quark number densities of the medium to be zero and leaving only a dependence on the isospin chemical potential. In the result of these simplifications the isospin medium is obtained for which the background geometry in the dual theory has no modification and the metric remains an ordinary AdS space $[47,48]$. Such a model is useful to separate the effects taking place due to isospin from the ones occurring under the influences of other quantities of the dense medium $[1,34,38,39]$. One of such effects is the splitting of the mass spectra of the meson states from the same isospin triplet in the medium due to their isospin interaction with the non-zero isospin of the medium. This effect was considered by a number of authors 
within the holographic QCD [30,34-36,47-51]. In [47,48] in the hard-wall model framework the meson mass splitting effect was considered for the triplets of the light mesons $\rho$, $\pi$, and $a_{1}$ in the dense nuclear matter due to the isospin interaction in connection with the study of the pion condensation in the isospin medium, which had been carried out in [34]. In this paper we reconsider this effect because of the recent observation of a relation between the definition of the effective mass for a meson in the medium with non-zero isospin and a fixed infrared boundary of the hardwall model. Here we define an effective mass following the standard definition in field theory and show that this definition will require fixing the IR boundary differently for the mesons from the same meson isotriplet in the medium. In other words the splitting of the meson mass leads to the splitting of the IR boundary of the model, since the mass spectrum in this model is directly defined by the value of the IR boundary. It should be noticed that the IR boundary shift in the hard-wall model already is known from Refs. [37,52], where the authors deal with the bulk interaction of the fields, which contribute to the boundary meson mass.

The paper is organized as follows: In Sect. 2 we give a description for the dense and isospin mediums in the hardwall model. In Sect. 3 we reproduce the equation of motion for the $\rho$ mesons in the isospin medium determine the effective mass of these mesons by means of holography. We avoid to distinguish the IR boundary introducing boundary terms. In Sect. 4 a similar analysis was made for the $\pi$ and $a_{1}$ mesons. We discuss the relation between the mass shift and the boundary terms in Sect. 5.

\section{Isospin medium in hard-wall model}

Let us present at first a bulk foundation for the boundary isospin medium following earlier work $[42,47,48,53]$. The bulk field content for the isospin medium will be a simplified version of the one for the dense nuclear medium utilized in Ref. [47]. Since this medium is described by the $S U$ (2) group, the bulk flavor gauge group should be chosen as $S U(2)_{L} \times S U(2)_{R}$ which then will be broken to $S U(2)_{V}$. We introduce in the bulk of AdS space two gauge fields $\mathcal{A}^{(L)}$ and $\mathcal{A}^{(R)}$ transforming under the $(1,0)$ and $(0,1)$ representations of this flavor symmetry group, respectively. The field strength tensor for these fields is

$\mathcal{F}_{M N}=\partial_{M} \mathcal{A}_{N}-\partial_{N} \mathcal{A}_{M}-i\left[\mathcal{A}_{M}, \mathcal{A}_{N}\right]$,

with

$\mathcal{A}_{M}=\mathcal{A}_{M}^{a} T^{a}$,

where the flavor index $a$ runs over $a=1,2,3$ and $T^{a}$ are the generators in the spinor representation of the $S U(2)_{L}$ or
$S U(2)_{R}$ group and are expressed in terms of Pauli matrices $\sigma^{a}$ by

$T^{a}=\frac{1}{2} \sigma^{a}$.

As a gauge condition on $\mathcal{A}_{z}$ we shall choose the axial gauge, $\mathcal{A}_{z}=0$.

The action for the gravity and for the gauge fields will be written as

$$
\begin{aligned}
S= & \int \mathrm{d}^{5} x \sqrt{-G}\left[\frac{1}{2 \kappa^{2}}(\mathcal{R}-2 \Lambda)\right. \\
& \left.-\frac{1}{4 g^{2}} \operatorname{Tr}\left(\mathcal{F}_{M N}^{(L)} \mathcal{F}^{(L) M N}+\mathcal{F}_{M N}^{(R)} \mathcal{F}^{(R) M N}\right)\right],
\end{aligned}
$$

where $\Lambda=-6 / R^{2}$ is the cosmological constant. The AdS/CFT correspondence establishes the following relations between the constants $\kappa^{2}, g^{2}$, the number of colors $N_{c}$, and the radius $R$ of space-time: $1 / g^{2}=N_{c} /\left(4 \pi^{2} R\right)$ and $1 /\left(2 \kappa^{2}\right)=N_{c}^{2} /\left(8 \pi^{2} R^{3}\right)$. The gauge fields $\mathcal{A}_{M}^{(L),(R)}$ are divided into the background parts $L_{M}, R_{M}$ and the fluctuations $l_{M}, r_{M}$ of these parts:

$\mathcal{A}_{M}^{(L)}=L_{M}+l_{M}$,

$\mathcal{A}_{M}^{(R)}=R_{M}+r_{M}$.

In the holographic description of the nuclear matter the bulk flavor group is $U(2)_{L} \times U(2)_{R}$ and this matter in the boundary QCD is given by the bulk gauge fields $L_{M}, R_{M}$, while the fluctuations $l_{M}, r_{M}$ in the bulk are needed for the description of the vector and axial-vector mesons in the boundary QCD. The homogeneous and isotropic matter at the boundary is described by the dual bulk fields $L_{M}$ and $R_{M}$, which are not dependent on the space-time coordinates. Moreover, the only time components $L_{0}$ and $R_{0}$ of these fields are taken non-zero, because the only flavor diagonal element of them $(a=3)$ corresponds to the physical quantity of the boundary matter. From these components we compose the vector and axial-vector fields:

$V_{0}^{3}=\frac{1}{2}\left(L_{0}^{3}+R_{0}^{3}\right)$,

$A_{0}^{3}=\frac{1}{2}\left(L_{0}^{3}-R_{0}^{3}\right)$.

The boundary value of $V_{0}^{3}$ maps to the isospin chemical potential of the medium $u$ and $d$ quarks $^{1}{ }^{\text {[53]. Following }}$ $[47,48]$ here we shall deal with the case $L_{0}^{a}=R_{0}^{a}$, which means Lagrangian invariance under changing the left and right flavor groups $S U(2)_{L} \leftrightarrow S U(2)_{R}$. Obviously, $A_{0}^{3}=0$ for this case. This $L \leftrightarrow R$ invariance in the dual boundary theory means that the medium particles, i.e. the nucleons, are required to be in the parity-even states. As is well known,

\footnotetext{
${ }^{1}$ In the confinement phase these quantities are with respect to the nucleons.
} 
the ground states of the nucleons are parity-even ones, and the first excited state of the nucleon can be a parity-even or a parity-odd state. In the parity-even state the nucleons have less energy than in the parity-odd one $[15,16]$.

The field strength tensor for $V_{0}^{3}$ is a flavor diagonal matrix as well and hence it gets the following form for an Abelian field:

$F_{M N}^{3}=\partial_{M} V_{N}^{3}-\partial_{N} V_{M}^{3}$.

In such a way the $S U(2)$ symmetry of the $V_{M}^{a}$ part of the gauge fields is broken down to two $U(1)$ symmetries. Meanwhile, the fluctuations $l_{M}, r_{M}$ remain non-abelian. Then the $V_{M}^{3}$ part of the action (2) gets the form

$S=\int \mathrm{d}^{5} x \sqrt{-G}\left[\frac{1}{2 \kappa^{2}}(\mathcal{R}-2 \Lambda)-\frac{1}{4 g^{2}} F_{M N}^{3} F^{3 M N}\right]$.

The holographic dual of the $A_{0}^{(u),(d)}= \pm \frac{1}{\sqrt{2}} V_{0}^{3}$ field will be the $u$-and $d$-quarks of the boundary medium. Imposing the hard-wall cut-off on the bulk radial coordinate $z$ makes these quarks confined ones in the boundary theory. It should be noted that the number densities of quarks thus defined (isoquarks) are zero (see [47]).

In the nuclear matter case the bulk geometry is a thermally charged AdS space (tcAdS) with radius $R$, which is the non-black brane solution of the Einstein-Maxwell system of equations obtained from (8) [42]:

$\mathrm{d} s^{2}=\frac{R^{2}}{z^{2}}\left(-f(z) \mathrm{d} t^{2}+\frac{1}{f(z)} \mathrm{d} z^{2}+\mathrm{d} \vec{x}^{2}\right)$.

Here

$f(z)=1+z^{6} \sum_{\alpha=u, d} q_{\alpha}^{2}$.

The constant $q_{\alpha}$ is related to the number densities $Q_{\alpha}$ of the medium $u$ and $d$ quarks, $q_{\alpha}=\sqrt{2} \kappa Q_{\alpha} /(\sqrt{3} g R)$. In the isospin medium case the number densities $Q_{\alpha}$ are taken zero and consequently the metric (9) returns into the metric of ordinary AdS space:

$\mathrm{d} s^{2}=\frac{R^{2}}{z^{2}}\left(-\mathrm{d} t^{2}+\mathrm{d} z^{2}+\mathrm{d} \vec{x}^{2}\right)$.

The radial coordinate $z$ ranges in the limited area $0<z \leq$ $z_{I R}$ due to the hard-wall cut-off.

It can be seen from a comparison of the geometries (9) and (11) in the dual boundary theory that there is an effect of the dense medium on the mesons which is provided by the $f(z)$ function in the bulk metric tensor $G^{M N}$. For instance, if the kinetic energy of the $l_{M}$ or $r_{M}$ field is $\mathcal{E}^{\prime}$ in the presence of medium fields $A_{0}^{(\alpha)}$ and is $\mathcal{E}$ in the absence of these medium fields, then $\mathcal{E}$ and $\mathcal{E}^{\prime}$ will be related by the relation $\mathcal{E}^{\prime}=G^{00} / G^{\prime 00} \mathcal{E}=f(z) \mathcal{E}$. Since in the isospin medium case $Q_{\alpha}=0$ and $f(z)=1$ there is no modification of the AdS geometry by the medium fields and the effect of the metric (11) on the $l_{M}$, and $r_{M}$ fields is the same as in free field case.

Solutions to the Maxwell equations obtained from the action (8) have the form

$A_{0}^{(\alpha)}=2 \pi^{2} \mu_{\alpha}-Q_{\alpha} z^{2}, \quad \alpha=u, d$.

In the isospin medium case the vector potential $A_{0}^{(\alpha)}$ is constant and is equal to the quark chemical potential $\mu_{\alpha}$ with a factor $2 \pi^{2}$ :

$A_{0}^{(\alpha)}=2 \pi^{2} \mu_{\alpha}$.

Here we are going to deal with the medium in the confining phase of the medium $u$ and $d$ quarks, where the fundamental excitations are nucleons but not quarks. So, the solution (13) should be expressed in terms of the chemical potentials of the nucleons. Taking into account the quark content of nucleons as in [47] the isospin chemical potentials of nucleons may be defined as a sum of quark chemical potentials $\mu_{P}=2 \mu_{u}+\mu_{d}$ and $\mu_{N}=\mu_{u}+2 \mu_{d}$. For isospin matter with two flavors the number densities of the nucleons are zero and $V_{0}^{3}$ equals $\sqrt{2} \pi^{2}\left(\mu_{u}-\mu_{d}\right)$ in the case of the deconfinement phase and $V_{0}^{3}=\sqrt{2} \pi^{2}\left(\mu_{P}-\mu_{N}\right)$

in the confinement phase one. Thus, $V_{0}^{3}$ in the dual boundary theory describes the homogeneous and constant isospin background field of a medium which is made of isonucleons.

\section{$3 \rho$ Meson mass splitting in the isospin medium}

\subsection{Effective mass definition by use of holography}

In this subsection applying the AdS/CFT correspondence we shall derive an effective mass formula for the vector meson minimally interacting with the external isospin field (14). It is well known that in AdS/QCD models the mesons in the dual boundary theory are described by the fluctuations of the gauge and scalar fields in the bulk. A bulk vector field fluctuation, which corresponds to the boundary vector meson, is composed of the left and right fluctuations $l_{\mu}^{i}$ and $r_{\mu}^{i}$ :

$v_{\mu}^{i}=\frac{1}{\sqrt{2}}\left(l_{\mu}^{i}+r_{\mu}^{i}\right)$,

where $\mu=0,1,2,3$ is for the boundary coordinates and $i=1,2,3$ is for the $S U(2)$ index. The fluctuations $l_{M}$ and $r_{M}$ transform under the fundamental representation of $S U(2)_{V}$ group $\left(l_{\mu}=l_{\mu}^{i} \frac{\sigma^{i}}{2}\right.$ and $\left.r_{\mu}=r_{\mu}^{i} \frac{\sigma^{i}}{2}\right)$. As a condition for gauge fixing of the fifth components of the $l_{M}$ and $r_{M}$ fields the axial gauge is chosen, which takes values $l_{z}^{i}=r_{z}^{i}=0$. The temporal component of the vector fluctuations will correspond to the fluctuations in the chemical potential (or the number density in the nuclear matter case) 
of medium particles. Setting this component zero $\left(v_{0}^{i}=0\right)$ we shall consider a medium with a constant isospin chemical potential. We suppose the bulk $v_{\mu}$ field does not depend on the spatial coordinates $x_{m}$. The action for the total vector field $\mathcal{V}_{M}=V_{M}+v_{M}=\frac{1}{\sqrt{2}}\left(L_{M}+R_{M}+l_{M}+r_{M}\right)$ has the form $[47,48]$

$S_{\mathcal{V}}=-\frac{1}{4 g^{2}} \int \mathrm{d}^{5} x \sqrt{-G} \operatorname{Tr}\left(\mathcal{F}_{M N}^{(\mathcal{V}) *} \mathcal{F}^{(\mathcal{V}) M N}\right)$

where $\mathcal{F}_{M N}^{\mathcal{V}}=\partial_{M} \mathcal{V}_{N}-\partial_{N} \mathcal{V}_{M}-i\left[\mathcal{V}_{M}, \mathcal{V}_{N}\right]$ is the field strength tensor. After taking the trace in (16) the action $S_{\mathcal{V}}$ gets the form

$$
\begin{aligned}
S_{\mathcal{V}}= & -\frac{1}{4 g^{2}} \int \mathrm{d}^{5} x \sqrt{-G}\left\{\sum _ { i = 1 } ^ { 3 } G ^ { \mu \nu } \left[G^{z z} \partial_{z} v_{\mu}^{i *} \partial_{z} v_{v}^{i}\right.\right. \\
& \left.+G^{m n} \partial_{\mu} v_{m}^{i *} \partial_{\nu} v_{n}^{i}\right]+G^{00} G^{m n} V_{0}^{3}\left[V_{0}^{3}\left(v_{m}^{1 *} v_{n}^{1}+v_{m}^{2 *} v_{n}^{2}\right)\right. \\
& \left.\left.+2\left(v_{m}^{1 *} \partial_{0} v_{n}^{2}-v_{m}^{2 *} \partial_{0} v_{n}^{1}\right)\right]\right\} .
\end{aligned}
$$

Here the $m, n=1,2,3$ indices denote the boundary spatial coordinates. The terms in the second square bracket in (17) describe the interaction of the vector field fluctuations with the background field $V_{0}^{3}$. In the dual boundary theory this interaction corresponds to the interaction of the vector meson with the isospin medium. Thus, the vector meson in the isospin medium in a holographic picture is described by the interacting gauge field theory in the bulk of AdS space.

The complex vector field $\rho_{m}$ is composed of the vector field components $v_{m}^{i}$ as follows:

$\rho_{m}^{0}=v_{m}^{3}$,

$\rho_{m}^{ \pm}=\frac{1}{\sqrt{2}}\left(v_{m}^{1} \pm i v_{m}^{2}\right)$.

As will be seen below the boundary values of the $\rho_{m}^{a}$ bulk fields correspond to the neutral and charged vector mesons, respectively. For the free bulk gauge field $\rho_{m}^{a}$ we can write the Fourier transformation:

$\rho_{m}^{0}(t, z)=\int \frac{\mathrm{d} \omega_{0}}{2 \pi} e^{-i \omega_{0} t} \rho_{m}^{0}\left(\omega_{0}, z\right)$

$\rho_{m}^{ \pm}(t, z)=\int \frac{\mathrm{d} \omega_{ \pm}}{2 \pi} e^{-i \omega_{ \pm} t} \rho_{m}^{ \pm}\left(\omega_{ \pm}, z\right)$

where the kinetic energies $\omega_{a}$ of the $\rho^{a}$ fields

$\partial_{0} \partial^{0} \rho_{n}^{a}=\omega_{a}^{2} \rho_{n}^{a} \quad(a=0, \pm)$

in the dual boundary theory correspond to the masses of the $\rho$ mesons. Equation (20) is the one defining a mass in the free field theory. In interacting gauge field theory, where the interaction is included by minimal coupling, the mass of the interacting field is defined by means of covariant derivatives instead of ordinary ones in (20). Also, as was mentioned above the background gauge field $V_{0}^{3}$ does not change the background geometry (11). So, the interaction with the $V_{0}^{3}$ field still was not included into Eqs. (19)-(20) and the kinetic energies $\omega_{ \pm}$in these formulas are the ones for the free $\rho^{a}$ field. As is well known, the vacuum masses of all $\rho$ mesons (charged and neutral ones) are equal. This degeneracy takes place for the masses $\omega_{a}$ of the bulk $\rho$ fields in the isospin background $V_{0}^{3}$ as well, since there is no metric change by this background:

$\omega_{+}=\omega_{-}=\omega_{0}$

From the action (17) written in terms of the $\rho^{a}$ fields after taking into account (21) we will obtain the following equations of motion:

$\partial_{z}\left(\sqrt{-G} G^{z z} G^{m n} \partial_{z} \rho_{n}^{0}\right)-\omega_{0}^{2} \sqrt{-G} G^{00} G^{m n} \rho_{n}^{0}=0$,

$\partial_{z}\left(\sqrt{-G} G^{z z} G^{m n} \partial_{z} \rho_{n}^{ \pm}\right)-\left(\omega_{ \pm}^{2}+\left(V_{0}^{3}\right)^{2} \mp 2 \omega_{ \pm} V_{0}^{3}\right)$

$\times \sqrt{-G} G^{00} G^{m n} \rho_{n}^{ \pm}=0$.

Explicitly, Eq. (22) will be written as below:

$\partial_{z}^{2} \rho_{n}^{0}-\frac{1}{z} \partial_{z} \rho_{n}^{0}+\omega_{0}^{2} \rho_{n}^{0}=0$

$\partial_{z}^{2} \rho_{n}^{ \pm}-\frac{1}{z} \partial_{z} \rho_{n}^{ \pm}+\left(\omega_{ \pm} \mp \sqrt{2} \pi^{2}\left(\mu_{P}-\mu_{N}\right)\right)^{2} \rho_{n}^{ \pm}=0$,

which have the same form as the ones obtained in [34] for the pions. These equations of motion were obtained in [48] introducing $v^{i}$ fields minimally interacting with the $V_{0}^{3}$ field. The Lagrangian for this system was constructed by means of the covariant derivative $\mathcal{D}_{M}$ as follows:

$L_{v_{M}^{i}+V_{0}^{3}}=-\frac{1}{4 g^{2}}\left(\mathcal{F}_{M N}^{i *} \mathcal{F}^{i M N}\right)$.

Here $\mathcal{F}_{M N}^{i}$ is defined as $\mathcal{F}_{M N}^{i}=\mathcal{D}_{M} v_{N}^{i}-\mathcal{D}_{N} v_{M}^{i}$ and the gauge covariant derivative $\mathcal{D}_{M}$ is $\mathcal{D}_{M} v_{N}^{i}=\partial_{M} v_{N}^{i}+$ $i\left(T^{i}\right)_{k j} V_{M}^{k} v_{N}^{j}=\partial_{M} v_{N}^{i}+\varepsilon^{i 3 j} V_{M}^{3} v_{N}^{j}$. The $\left(T^{i}\right)_{k j}=-i \varepsilon^{i k j}$ are the generators of the isospin group in the adjoint representation. Taking into account the transverseness of the $v^{i M}$ field $\mathcal{D}_{M} v^{i M}=0$ in (24) the action for this Lagrangian will be reduced to the following form:

$S_{v^{i}+V_{0}^{3}}=-\frac{1}{4 g^{2}} \int \mathrm{d}^{5} x \sqrt{-G}\left(\mathcal{D}_{M} v^{i N}\right)^{*}\left(\mathcal{D}^{M} v_{N}^{i}\right)$.

This action in terms of the $\rho$ fields will split into three independent terms for each $\rho^{a}$ component:

$S_{\rho_{n}^{a}+V_{0}^{3}}=-\frac{1}{4 g^{2}} \int \mathrm{d}^{5} x \sqrt{-G} \sum_{a=0, \pm}\left(D_{M}^{(a)} \rho^{a n}\right)^{*}\left(D^{(a) M} \rho_{n}^{a}\right)$.

Here the covariant derivative $D_{M}^{(a)}$ for the $\rho^{a}$ field has components $D_{\mu}^{(a)}=\partial_{\mu}+i e^{(a)} V_{0}^{3}, D_{z}^{(a)}=\partial_{z}$ with $e^{(a)}= \pm 1,0$. In the action (26) as in Eq. (18), the terms of cubic and fourth 
order of the fluctuations were neglected. The equation of motion obtained from the action (26) has the form

$D_{M}^{(a)}\left(\sqrt{-G} G^{M M^{\prime}} G^{m n} D_{M^{\prime}}^{(a)} \rho_{n}^{a}\right)=0$.

This is a five-dimensional D'Alembert equation in curved space-time (11) for the vector field having a zero fifth component $\left(\rho_{5}=0\right)$ and zero five-dimensional mass $\left(m_{5}=0\right)$. Boundary terms which arise on obtaining (27) lead to the boundary conditions $\left.\partial_{z} \rho_{n}^{a}\right|_{z_{I R}}=0$ and $\left.\rho_{n}^{a}(z)\right|_{\varepsilon}=0$, which are the same as the ones in the free field case. The explicit form of Eq. (27) is Eq. (22).

It is useful to rewrite Eq. (27) as a sum of its fourdimensional part and the $z$ coordinate part

$$
\begin{aligned}
& \frac{1}{\sqrt{-G}} \partial_{z}\left(\sqrt{-G} G^{z z^{\prime}} G^{m n} \partial_{z^{\prime}} \rho_{n}^{a}\right) \\
& +\frac{1}{\sqrt{-G}} D_{\mu}^{(a)}\left(\sqrt{-G} G^{\mu \mu^{\prime}} G^{m n} D_{\mu^{\prime}}^{(a)} \rho_{n}^{a}\right)=0,
\end{aligned}
$$

where we divided it by $\sqrt{-G}$. Now, let us write the D'Alembert equation in a four-dimensional curved spacetime for the massive vector field $\rho_{n}^{a}$ minimally coupled with the constant external background gauge field $V_{\mu}^{a}$ :

$$
\frac{1}{\sqrt{-G}} D_{\mu}^{(a)}\left(\sqrt{-G} G^{\mu \mu^{\prime}} G^{m n} D_{\mu^{\prime}}^{(a)} \rho_{n}^{a}\right)-\left(m_{a}^{*}\right)^{2} G^{m n} \rho_{n}^{a}=0,
$$

where the $m_{a}^{*}$ is the mass of the $\rho_{n}^{a}$ field. In our case the four-dimensional space-time is the UV boundary of the AdS space (11) and the massive vector field is the boundary value of the $\rho$ field. In the AdS/CFT correspondence here the UV values of the $\rho^{a}$ fields correspond to the $\rho$ mesons defined on this boundary and the equations of motion for these fields at the UV boundary correspond to the equations of motion for these mesons. Hence, we have to correlate Eq. (28), the equations in the UV limit, to the ones in (29). From this correspondence it is seen that the eigenvalues of the $-\frac{1}{\sqrt{-G} G^{m n}} \partial_{z}\left(\sqrt{-G} G^{z z^{\prime}} G^{m n} \partial_{z^{\prime}}\right)$ operator correspond to the squared mass $\left(m_{a}^{*}\right)^{2}$ of the $\rho^{a}$ vector fields in (29). So, we may accept the equality of the eigenvalues of this operator to the squared masses of boundary meson fields:

$$
-\frac{1}{\sqrt{-G} G^{m n}} \partial_{z}\left(\sqrt{-G} G^{z z^{\prime}} G^{m n} \partial_{z^{\prime}} \rho_{n}^{a}\right)=\left(m_{a}^{*}\right)^{2} \rho_{n}^{a} .
$$

Equation (30) is the determining formula of the effective mass for the vector field in the isospin medium by means of the derivative operator over the extra dimension.

In the AdS space geometry (11) the equations of motion (28) have got the form

$\partial_{z}\left(\frac{1}{z} \partial_{z} \rho_{n}^{a}\right)+\frac{1}{z} D_{\mu}^{(a)} D^{(a) \mu} \rho_{n}^{a}=0$, which is the same as (23). The operator $D_{\mu}^{(a)} D^{(a) \mu}$ has the following eigenvalues:

$$
\begin{aligned}
& D_{\mu}^{(0)} D^{(0) \mu} \rho_{n}^{0}=\omega_{0}^{2} \rho^{0}=\left(m_{0}^{*}\right)^{2} \rho_{n}^{0}, \\
& \begin{aligned}
D_{\mu}^{( \pm)} D^{( \pm) \mu} \rho_{n}^{ \pm} & =\left(\omega_{ \pm} \mp \sqrt{2} \pi^{2}\left(\mu_{P}-\mu_{N}\right)\right)^{2} \rho_{n}^{ \pm} \\
& =\left(m_{ \pm}^{*}\right)^{2} \rho_{n}^{ \pm} .
\end{aligned}
\end{aligned}
$$

In four-dimensional space-time, where Eq. (29) was written, the eigenvalues (32) determine the effective masses of the massive $\rho^{a}$ fields:

$D_{\mu}^{(a)} D^{(a) \mu} \rho_{n}^{a}=\left(m_{a}^{*}\right)^{2} \rho_{n}^{a}$,

according to the effective mass definition of vector field in the constant background field in four-dimensional gauge field theory. The two definitions of the effective mass (30) and (33) give same result and thus, the masses $m_{a}^{*}$ are the masses of the $\rho^{a}$ mesons in the isospin medium.

\subsection{Effective mass as an eigenvalue of profile function}

Another way of determining the effective mass of mesons is using profile function solution. From the two-point correlation function study it is well known that the eigenvalues in the profile function are the mass spectrum of the vector meson in the vacuum case [54]. It is natural to extend this study to the constant isospin medium case. To this end let us write a solution to Eq. (23) for the $\rho_{a}^{s}$ mode in the Kaluza-Klein decomposition $\rho^{a}\left(p^{0}, z\right)=\sum_{s} r_{a}^{s}\left(\mathbf{m}_{a}^{s}\right) \rho_{a}^{s}\left(\mathbf{m}_{a}^{s}, z\right)$ :

$$
\begin{aligned}
\rho_{a}^{s}\left(\mathbf{m}_{a}^{s}, z\right)= & \mathbf{m}_{a}^{s} z\left[Y_{0}\left(\mathbf{m}_{a}^{s} z_{I R}\right) J_{1}\left(\mathbf{m}_{a}^{s} z\right)\right. \\
& \left.-J_{0}\left(\mathbf{m}_{a}^{s} z\right) Y_{1}\left(\mathbf{m}_{a}^{s} z_{I R}\right)\right] .
\end{aligned}
$$

Here $\mathbf{m}_{a}^{s}$ denotes the square root of the bracket in Eq. (23) for the $s$ mode:

$\mathbf{m}_{0}^{s}=\omega_{0}^{s}$,

$\mathbf{m}_{ \pm}^{s}=\omega_{0}^{s} \mp V_{0}^{3}$,

and $\omega_{a}^{s}$ is the mass of the free $s$ mode.

This solution is the same as the one found in [54] for the free vector field $V_{\mu}$, except for the constant eigenvalues $\mathbf{m}_{a}^{s}$ in the arguments of the Bessel function, which were denoted by $M_{n}$ in the free vector field case. Of course, the distinction between the $M_{n}$ and $\mathbf{m}_{a}^{s}$ constants will not change the procedure of finding the poles by means of the two-point function

$$
\int \mathrm{d}^{4} x e^{i p x}\left\langle J_{\mu}^{a}(x) J_{v}^{b}(0)\right\rangle=\delta^{a b}\left(\eta_{\mu \nu}-\frac{p_{\mu} p_{v}}{p^{2}}\right) \Sigma\left(p^{2}\right) .
$$

Here $\Sigma\left(p^{2}\right)$ was defined by means of the profile function $V(p, z)$ of the vector field as follows:

$$
\Sigma\left(p^{2}\right)=-\frac{1}{g_{5}^{2}}\left(\frac{1}{z V(p, z)} \frac{\partial V(p, z)}{\partial z}\right)_{z=0} .
$$


In the free field case $V(p, z)$ has the form of $\rho_{s}^{a}\left(\omega, V_{0}^{3}, z\right)$ at $V_{0}^{3}=0$ in (34) and for this solution $\Sigma\left(p^{2}\right)$ gets the form [54]

$\Sigma\left(p^{2}\right)=\frac{\pi p^{2}}{2 g_{5}^{2}}\left[Y_{0}(p z)-J_{0}(p z) \frac{Y_{0}\left(p z_{I R}\right)}{J_{0}\left(p^{2} z_{I R}\right)}\right]$.

Applying a Kneser-Sommerfeld expansion to Eq. (38) in [54] an explicit pole structure of the $\Sigma\left(p^{2}\right)$ was found:

$\Sigma\left(p^{2}\right)=\frac{2 p^{2}}{g_{5}^{2} z_{I R}^{2}} \sum_{n=1}^{\infty} \frac{1}{\left[J_{1}\left(\gamma_{0}^{S}\right)\right]^{2}\left[p^{2}-\left(M_{n}\right)^{2}\right]}$,

where $\gamma_{0}^{s}$ are the zeros of the Bessel function $J_{0}(z)$. For the solution (34) the $\Sigma\left(p^{2}\right)$ in (39) will have the same expression with the replacement of $M_{n}$ by $\mathbf{m}_{a}^{s}$ in it and we shall get poles at $p_{0}^{2}=\left(\mathbf{m}_{a}^{s}\right)^{2}$. Since the poles of the two-point function are the masses of the vector mesons in dual theory the poles $\mathbf{m}_{a}^{s}$ will be effective masses of these mesons in the isospin medium. The constants $\mathbf{m}_{a}^{s}$ are the eigenvalues of both the $D_{\mu}^{(a)} D^{(a) \mu}$ and the $-z \partial_{z}\left(\frac{1}{z} \partial_{z} \rho_{n}^{a}\right)$ operators for the $s$ mode according to (33) and (31).

Thus, two ways of determination of the effective masses give the same result and the effective mass of the dual $\rho$ mesons in the isospin medium are the following ones:

$m_{ \pm}^{*}=\left|\omega_{ \pm} \mp V_{0}^{3}\right|$,

$m_{0}^{*}=\omega_{0}$.

Although the vacuum masses $\omega_{a}$ of $\rho$ mesons are equal, their in-medium masses $m_{a}^{*}$ are different. The splitting in the mass spectrum of $\rho$ mesons occur due to interaction with the isospin medium. After taking into account in (40) the equality of the vacuum mass $\left(\omega_{ \pm}=\omega_{0}\right)$, the splitting formula will look like

$m_{ \pm}^{*}=\left|m_{0}^{*} \mp V_{0}^{3}\right|=\left|m_{0}^{*} \mp \sqrt{2} \pi^{2}\left(\mu_{P}-\mu_{N}\right)\right|$.

The mass splitting formula (41) shows a decreasing of the mass for the positively charged meson and increasing it for the negatively charged one when the positive difference of chemical potentials $\mu_{P}-\mu_{N}$ grows, and vise versa for the negative $\mu_{P}-\mu_{N}$. The isospin medium eliminates the isospin degeneration the of mass, and degeneration occurs when the chemical potentials of the medium protons and neutrons are equal.

The splitting of the meson mass in the holographic approach was studied in $[34,35,47,48]$, but the sign of the splitting obtained (41) is different from the one obtained in $[34,47,48]$,

$m_{0}^{* *}=\omega_{0}$,

$m_{ \pm}^{\prime *}=\omega_{ \pm} \pm V_{0}^{3}=m_{0}^{\prime *} \pm \sqrt{2} \pi^{2}\left(\mu_{P}-\mu_{N}\right)$,

and it agrees with the one obtained in $[35,55]$ if we take the value of $V_{0}^{3}$ in (41) equal to the $\mu_{I}$ introduced in [35]. It is obvious that the effective mass formula (42) does not obey the holographic definition (30). This distinction between Eqs. (41) and (42) is connected with the definition of the effective mass and will be clarified in the next subsection. The sign of the splitting has importance for meson condensation [55]. According to (41) the positively charged meson becomes massless at a positive value of the isospin medium, it condenses at the value $V_{0}^{3}=\omega_{+}$, while a condensation of the negatively charged meson takes place at $\omega_{-}=-V_{0}^{3}$.

\subsection{Mass spectrum and boundary terms}

Applying the Neumann boundary condition at the IR boundary $\left.\partial_{z} \rho^{a}(z)\right|_{z_{I R}}=0$ on solution (34) yields the following formula [52] for the mass spectra $m_{a}^{s}$ :

$$
\begin{aligned}
& \mathbf{m}_{0}^{s} \simeq\left(s-\frac{1}{4}\right) \frac{\pi}{z_{I R}^{0}}, \\
& \mathbf{m}_{ \pm}^{s} \simeq\left(s-\frac{1}{4}\right) \frac{\pi}{z_{I R}^{ \pm}} \quad s=1,2,3 \ldots
\end{aligned}
$$

which has same form as one in the vacuum and corresponds to mass spectra of excited states of the $\rho$ mesons in the medium. Here we have distinguished the values of the IR boundary for the differently charged meson, denoting them $z_{I R}^{0}$ and $z_{I R}^{ \pm}$, respectively. Otherwise, the equality of the right hand sides of the first and second equalities in (43), $\left(\left(s-\frac{1}{4}\right) \pi / z_{I R}^{ \pm}=\right.$ $\left.\left(s-\frac{1}{4}\right) \pi / z_{I R}^{0}\right)$ would mean the equality of their left hand sides also, $\left(\omega_{0}^{s} \mp \sqrt{2} \pi^{2}\left(\mu_{P}-\mu_{N}\right)=\omega_{0}^{s}\right)$, which is nonsense in the non-zero medium isospin case. However, when we introduce different $z_{I R}$ for different components, we get an interaction of fields defined in different space-times, which is not appropriate as well. In order to solve this dilemma we can add to the action new boundary terms, which will lead to the same Kaluza-Klein spectrum (43) for all $\rho^{a}$ components and therefore will ensure fixing $z_{I R}^{ \pm}$at the same value with $z_{I R}^{0}$. Obviously, new boundary terms should have a form not changing the equation of motion (22) and (23). We introduce such a kind of boundary terms at $z=z_{I R}$ in the following form:

$S_{ \pm}= \pm V_{0}^{3} \int \mathrm{d}^{4} x \sqrt{-G} G^{00} G^{m n}\left(\left(\rho_{m}^{ \pm}\right)^{*} \rho_{n}^{ \pm}\right)_{z=z_{I R}}$.

In terms of the $v_{\mu}$ fields, $S_{+}$and $S_{-}$have the same expression with opposite sign:

$$
S_{ \pm}= \pm \frac{1}{2} V_{0}^{3} \int \mathrm{d}^{4} x \sqrt{-G} G^{00} G^{m n}\left(v_{m}^{1 *} v_{n}^{1}+v_{m}^{2 *} v_{n}^{2}\right)_{z=z_{I R}} .
$$

Then the total action for the $\rho^{a}$ fields will have the form

$$
S_{\rho+V_{0}^{3}}=\int \mathrm{d}^{4} x \int_{0}^{z_{I R}} \mathrm{~d} z \sqrt{-G} \sum_{a} \mathcal{L}^{(a)}+S_{+}+S_{-},
$$


where $\mathcal{L}^{(a)}$ is the Lagrangian term producing the equation of motion (22) for the $a$ isocomponent:

$$
\begin{aligned}
\mathcal{L}^{(0)}= & G^{z z} G^{m n}\left(\partial_{z}\left(\rho_{m}^{0}\right)^{*}\right)\left(\partial_{z} \rho_{n}^{0}\right)+\omega_{0}^{2} G^{00} G^{m n}\left(\rho_{m}^{0}\right)^{*} \rho_{n}^{0}, \\
\mathcal{L}^{( \pm)}= & G^{z z} G^{m n}\left(\partial_{z}\left(\rho_{m}^{ \pm}\right)^{*}\right)\left(\partial_{z} \rho_{n}^{ \pm}\right)+\left(\omega_{ \pm}^{2}+\left(V_{0}^{3}\right)^{2}\right. \\
& \left.\mp 2 \omega_{ \pm} V_{0}^{3}\right) G^{00} G^{m n}\left(\rho_{m}^{ \pm}\right)^{*} \rho_{n}^{ \pm} .
\end{aligned}
$$

When we derive the equations of motion (22) from the (47) the boundary terms arising on integration by parts have the same form $\left.\left(\rho^{a}\right)^{*} \partial_{z} \rho^{a}\right|_{z_{U V}} ^{z_{I R}}$ for the different $\rho^{a}$ components. These boundary terms will be summed with the $S_{ \pm}$boundary terms and the total boundary condition imposed on the $\rho^{a}$ solutions at the IR boundary ultimately will be written as below:

$$
\begin{aligned}
& \left.\left(\partial_{z} \rho^{0}(z)\right)\right|_{z_{I R}}=0,\left.\quad\left(\partial_{z} \rho^{+}(z)+V_{0}^{3} \rho^{+}(z)\right)\right|_{z_{I R}}=0, \\
& \left.\left(\partial_{z} \rho^{-}(z)-V_{0}^{3} \rho^{-}(z)\right)\right|_{z_{I R}}=0 .
\end{aligned}
$$

All three boundary conditions give the same quantization formula - the same spectrum (43) but for the vacuum masses $\omega_{0}^{s}$ of the in-medium $\rho$ mesons:

$$
\begin{aligned}
& \mathbf{m}_{0}^{s}=\omega_{0}^{s} \simeq\left(s-\frac{1}{4}\right) \frac{\pi}{z_{I R}} \\
& \mathbf{m}_{ \pm}^{s} \pm V_{0}^{3}=\omega_{ \pm}^{s} \mp V_{0}^{3} \pm V_{0}^{3}=\omega_{0}^{s} \\
& \simeq\left(s-\frac{1}{4}\right) \frac{\pi}{z_{I R}} \quad s=1,2,3 \ldots
\end{aligned}
$$

and the same $z_{I R}$ will be chosen in all three quantization formulas (49). Thus, we conclude that only the vacuum mass part $\omega_{0}^{s}$ of the effective mass of the meson is subject to quantization.

Now we can explain why there are two ways for the definition of the meson mass splitting (Eqs. (41) and (42)). The splitting formula (42) was obtained in the hard-wall model, which has one IR boundary for all isocomponents of the vector field. In this case all three equations in (23) were solved under the same boundary condition at IR and with the same value of $z_{I R}$. This dictates that all coefficients in front of the last terms in Eq. (23) should be the same. This leads to the splitting formula (42). In fact, if we put back (42) into (23) we shall get the same expression for these coefficients, which equals $\omega_{0}$, i.e. we have the same three equations for $\rho^{ \pm}$and $\rho^{0}$. Obviously, in this case all $\mathbf{m}_{a}^{s}$ coefficients in the Bessel function solutions are the same, $\mathbf{m}_{a}^{s}=\omega_{0}$, and the necessity does not arise to distinguish $z_{I R}$ in the mass spectrum formula (43). In (44) we obtained this spectrum formula introducing boundary terms, but the effective mass in our case obeys the defining relations (30) and (33).

The first state $(s=1)$ of the charged vector field tower (40) is the ground state of the $\rho^{ \pm}$meson, which has a mass

$$
\begin{aligned}
m_{\rho^{ \pm}} & \approx \frac{3}{4} \pi \frac{1}{z_{I R}^{0}} \mp \sqrt{2} \pi^{2}\left(\mu_{P}-\mu_{N}\right) \\
& \approx 2.4 z_{I R}^{-1} \mp \sqrt{2} \pi^{2}\left(\mu_{P}-\mu_{N}\right),
\end{aligned}
$$

and for the neutral $\rho$ meson we have the value of the mass in vacuum [52]:

$m_{\rho^{0}} \approx \frac{2.4}{z_{I R}^{0}}$.

Finally, the normalized solution (34) for the $\rho^{a}$ meson attains the same form as the one in the vacuum case [52]:

$\rho_{a}^{s}=\frac{z J_{1}\left(\mathbf{m}_{a}^{s} z\right)}{\sqrt{\int_{0}^{z_{I R}} \mathrm{~d} z z\left[J_{1}\left(\mathbf{m}_{a}^{s} z\right)\right]^{2}}}$.

\section{Mass splitting formula for the $a_{1}$ and $\pi$ mesons}

In this section we shall define the effective mass for the axialvector and pion fields using the holographic correspondence as was done for the $\rho$ mesons. Let us briefly present here the action and the equations of motion for the axial-vector and pion fields, all details of which can be found in $[47,48]$.

The non-zero fluctuations of the axial-vector field, which is defined as $a_{\mu}^{i}=\frac{1}{\sqrt{2}}\left(l_{\mu}^{i}-r_{\mu}^{i}\right)$, were introduced into the model in order to describe the axial-vector mesons in the boundary QCD. In addition, a complex scalar field $\Phi$ is introduced, which performs the chiral symmetry breaking $S U(2)_{L} \times S U(2)_{R} \rightarrow S U(2)_{V}$ of the model. The action for the $\Phi$ field has the form

$S_{\phi}=-\int \mathrm{d}^{5} x \sqrt{-G} \operatorname{Tr}\left[|D \Phi|^{2}+m_{5}^{2}|\Phi|^{2}\right]$.

Here $D_{M} \Phi=\partial_{M} \Phi-i L_{M} \Phi+i \Phi R_{M}$ and $m_{5}^{2}=-3$. The $\Phi$ field is written in the form $\Phi=\phi \exp \left[i \sqrt{2} \pi^{a} T^{a}\right]$. The fluctuations $\pi^{i}$ describe the pions in the dual theory. In the isospin medium case, as was mentioned, the background geometry is given by (11), and thus the solution for the $\phi$ field has the simple form of the one in the vacuum case:

$\phi(z)=\frac{1}{2} m_{q} z+\frac{1}{2} \sigma z^{3}$,

where $m_{q}$ is the mass of the light quarks and $\sigma$ is the value of the condensate. The action (53) contains an interaction of $\Phi$ field with the axial-vector field $a_{\mu}^{i}$ and the total action of these fields will be the sum of $S_{\phi}$ and the action $S_{a}$ for the fluctuations $a_{\mu}^{i}$. The action $S_{a}$, which describes the axialvector field interacting with the gauge field $V_{0}^{3}$ is obtained from the action (4) and has the form

$S_{a}=-\frac{1}{4 g^{2}} \int \mathrm{d}^{5} x \sqrt{-G}\left(\mathcal{F}_{M N}^{a *} \mathcal{F}^{a M N}\right)$,

where $\mathcal{F}_{M N}^{i}=\mathcal{D}_{M} a_{N}^{i}-\mathcal{D}_{N} a_{M}^{i}$ and the gauge covariant derivative $\mathcal{D}_{M}, \mathcal{D}_{M} a_{N}^{i}=\partial_{M} a_{N}^{i}+i\left(T^{i}\right)_{k j} V_{M}^{k} a_{N}^{j}=$ $\partial_{M} a_{N}^{i}+\varepsilon^{i 3 j} V_{M}^{3} a_{N}^{j}$, was defined as one for the vector field fluctuations $v^{i}$. Since in the dual QCD theory the axial-vector $a_{1}$ mesons are described by the transverse field we divide 
the bulk axial-vector field $a_{\mu}^{i}$ into the transverse $\bar{a}_{\mu}^{i}$ and the longitudinal $\chi$ parts: $a_{\mu}^{i}=\bar{a}_{\mu}^{i}+\partial_{\mu} \chi^{i} \quad\left(\partial^{\mu} \bar{a}_{\mu}=0\right)$. The longitudinal parts of the left and right fluctuations $l_{\mu}^{i}$ and $r_{\mu}^{i}$ are equal to $+\frac{1}{2} \partial_{\mu} \chi^{i}$ and $-\frac{1}{2} \partial_{\mu} \chi^{i}$, respectively, and the vector field fluctuations $v_{\mu}^{i}$ turn out to be transverse. In an equivalent way an action for the transverse axial-vector fluctuations $\bar{a}_{\mu}^{i}$ can be obtained from the action (4) and has the form [47]

$$
\begin{aligned}
S_{\bar{a}}= & -\frac{1}{4 g^{2}} \int \mathrm{d}^{5} x \sqrt{-G} G^{m n}\left\{\sum _ { i = 1 } ^ { 3 } \left[G^{z z} \partial_{z} \bar{a}_{m}^{i *} \partial_{z} \bar{a}_{n}^{i}\right.\right. \\
& \left.+G^{\mu \nu} \partial_{\mu} \bar{a}_{m}^{i *} \partial_{\nu} \bar{a}_{n}^{i}\right]+G^{00} V_{0}^{3}\left[V_{0}^{3}\left(\bar{a}_{m}^{1 *} \bar{a}_{n}^{1}+\bar{a}_{m}^{2 *} \bar{a}_{n}^{2}\right)\right. \\
& \left.\left.+2 G^{00}\left(\bar{a}_{m}^{1 *} \partial_{0} \bar{a}_{n}^{2}-\bar{a}_{m}^{2 *} \partial_{0} \bar{a}_{n}^{1}\right)\right]\right\} .
\end{aligned}
$$

The two actions (55) and (56) lead to the same equations of motion for the $\bar{a}$ field, as in the vector field case. It is recommended to incorporate the action for the longitudinal $\chi$ field with the action for the pseudoscalar field $\pi$ derived from (53). In summary the action of $\chi$ and $\pi$ fields has the following explicit form [47]:

$$
\begin{aligned}
& S_{\pi+\chi}=-\frac{1}{4 g^{2}} \int \mathrm{d}^{5} x \sqrt{-G}\left\{\sum_{i=1}^{3}\left[G^{z z} G^{\mu \nu} \partial_{z} \partial_{\mu} \chi^{i *} \partial_{z} \partial_{\nu} \chi^{i}\right]\right. \\
& +4 g^{2} \phi^{2}\left[\sum _ { i = 1 } ^ { 3 } \left(G^{\mu \nu} \partial_{\mu} \chi^{i *} \partial_{\nu} \chi^{i}+G^{z z} \partial_{z} \pi^{i *} \partial_{z} \pi^{i}\right.\right. \\
& \left.+G^{\mu \nu} \partial_{\mu} \pi^{i *} \partial_{\nu} \pi^{i}-2 G^{\mu \nu} \partial_{\mu} \chi^{i *} \partial_{\nu} \pi^{i}+G^{m n} \bar{a}_{m}^{i *} \bar{a}_{n}^{i}\right) \\
& +G^{00} V_{0}^{3}\left[2\left(\pi^{1 *} \partial_{0} \pi^{2}-\pi^{2 *} \partial_{0} \pi^{1}\right)+V_{0}^{3}\left(\left|\pi^{1}\right|^{2}+\left|\pi^{2}\right|^{2}\right)\right. \\
& \left.\left.\left.-2\left(\pi^{1 *} \partial_{0} \chi^{2}-\pi^{2 *} \partial_{0} \chi^{1}\right)\right]\right]\right\}
\end{aligned}
$$

This action includes the interaction term of the $\bar{a}^{i}$ field with the $\phi$ field, which is coming from the action (53) and obviously, this term will be present in the equation of motion for the $a_{1}$ field.

\subsection{The $a_{1}$ mesons}

Let us introduce the neutral $a_{1 m}^{0}$ and the charged $a_{1 m}^{ \pm}$ components for the axial-vector field $\bar{a}_{m}$, which will correspond to the respective axial-vector $a_{1}$ meson in the holography:

$$
\begin{aligned}
& a_{1 m}^{0}=\bar{a}_{m}^{3}, \\
& a_{1 m}^{ \pm}=\frac{1}{\sqrt{2}}\left(\bar{a}_{m}^{1} \pm i \bar{a}_{m}^{2}\right) .
\end{aligned}
$$

The Fourier transforms of these components are

$a_{1 m}^{0}(t, z)=\int \frac{\mathrm{d} \bar{\omega}_{0}}{2 \pi} e^{-i \bar{\omega}_{0} t} a_{1 m}^{0}\left(\bar{\omega}_{0}, z\right)$,

$a_{1 m}^{ \pm}(t, z)=\int \frac{\mathrm{d} \bar{\omega}_{ \pm}}{2 \pi} e^{-i \bar{\omega}_{ \pm} t} a_{1 m}^{ \pm}\left(\bar{\omega}_{ \pm}, z\right)$,

where $\bar{\omega}_{0}$ and $\bar{\omega}_{ \pm}$are the masses of free $a_{1}$ fields. The action for the $a_{1}^{a}$ field will be written in the form

$$
\begin{aligned}
S_{a_{1}^{a}+V_{0}^{3}}= & -\frac{1}{4 g^{2}} \int \mathrm{d}^{5} x \sqrt{-G} \sum_{a=0, \pm}\left\{\left(D_{M}^{(a)} a_{1}^{a}\right)^{*}\right. \\
& \left.\times\left(D^{(a) M} a_{1 n}^{a}\right)+4 g^{2} \phi^{2} a_{1}^{a}{ }^{n} a_{1 n}^{a}\right\},
\end{aligned}
$$

where the covariant derivative $D_{M}^{(a)}$ is the same as in the vector field case. The equations of motion for this field written in terms of $D_{M}^{(a)}$ will be obtained from the action (60) and has the form below:

$$
\begin{aligned}
& D_{M}^{(a)}\left(\sqrt{-G} G^{M M^{\prime}} G^{m n} D_{M^{\prime}}^{(a)} a_{1 n}^{a}\right)+4 g^{2} \phi^{2} \sqrt{-G} G^{m n} a_{1 n}^{a} \\
& =0 .
\end{aligned}
$$

This is a five-dimensional D'Alembert equation for the axialvector field interacting with the $\phi(z)$ field. Obviously, the fifth component and the five-dimensional mass $M_{5}$ of this field are taken to be zero. The boundary conditions, which will be imposed on $a_{1 n}$ are obtained from the boundary terms arising on deriving Eq. (61) and are the usual ones: $\left.\partial_{z} a_{1 n}^{a}\right|_{z_{I R}}=0$ and $\left.a_{1 n}^{a}(z)\right|_{\varepsilon}=0$.

It will be useful to rewrite Eq. (61) as the sum of its fourdimensional part and the fifth dimension part:

$$
\begin{aligned}
& \frac{1}{\sqrt{-G}} \partial_{z}\left(\sqrt{-G} G^{z z^{\prime}} G^{m n} \partial_{z^{\prime}} a_{1 n}^{a}\right)+\frac{1}{\sqrt{-G}} D_{\mu}^{(a)} \\
& \times\left(\sqrt{-G} G^{\mu \mu^{\prime}} G^{m n} D_{\mu^{\prime}}^{(a)} a_{1 n}^{a}\right)+4 g^{2} \phi^{2} G^{m n} a_{1 n}^{a}=0 .
\end{aligned}
$$

On the QCD side there is a massive axial-vector meson, which is described by the $a_{1 n}$ field and has a shift in the mass due to the quark condensate background. We may take into account this mass shift by adding to the QCD Lagrangian an additional mass term proportional to the value of condensate, which will be denoted by $4 g^{2} \phi_{0}^{2}$. The constant isospin background of the medium can be taken into account by introducing a constant gauge field $V_{0}^{3}$. The interaction with this background will be taken into account by the minimal interaction. Let us write the D'Alembert equation for such an $a_{1 n}$ field in a four-dimensional curved space-time, which is, in fact, the UV boundary of the AdS space (11):

$$
\begin{aligned}
& \frac{1}{\sqrt{-G}} D_{\mu}^{(a)}\left(\sqrt{-G} G^{\mu \mu^{\prime}} G^{m n} D_{\mu^{\prime}}^{(a)} a_{1 n}^{a}\right) \\
& +4 g^{2} \phi_{0}^{2} G^{m n} a_{1 n}^{a}-\left(\bar{m}^{a}\right)^{2} G^{m n} a_{1 n}^{a}=0 .
\end{aligned}
$$

Here $\bar{m}^{a}$ is the mass of the $a_{1 n}^{a}$ field not including the condensate shift and $\phi_{0}$ is the value of the $\phi(z)$ field at some fixed value of $z$. Since the AdS/CFT correspondence of the 
fields takes place at the UV boundary we take as a fixed value of $z$ the $z_{U V}$, i.e. $\phi_{0}=\phi\left(z_{U V}\right)$. The condensate interaction term $4 g^{2} \phi_{0}^{2} G^{m n} a_{1 n}^{a}$ in (63) could be included into the mass term by $m_{a}^{2}=\left(\bar{m}^{a}\right)^{2}-4 g^{2} \phi_{0}^{2}$. Correlating Eqs. (62) to (63) we observe the equality of the mass term in (62) and the z-dependent part of Eq. (63),

$\left(\bar{m}^{a}\right)^{2} G^{m n} a_{1 n}^{a}=-\frac{1}{\sqrt{-G}} \partial_{z}\left(\sqrt{-G} G^{z z^{\prime}} G^{m n} \partial_{z^{\prime}} a_{1 n}^{a}\right)$.

Equation (64) is the formula determining the effective mass of the axial-vector meson in the isospin medium by means of the derivative operator over the extra dimension defined in dual bulk theory. The equations of motion for the $a_{1}^{a}$ field are obtained from the summary action of (63) and (64) and given by [47]

$$
\begin{aligned}
& \partial_{z}\left(\sqrt{-G} G^{z z} G^{m n} \partial_{z} a_{1 n}^{0}\right)-\left(G^{00} \bar{\omega}_{0}^{2}+4 g^{2} \phi^{2}\right) \\
& \quad \times \sqrt{-G} G^{m n} a_{1 n}^{0}=0, \\
& \partial_{z}\left(\sqrt{-G} G^{z z} G^{m n} \partial_{z} a_{1 n}^{ \pm}\right)-\left[\left(\bar{\omega}_{ \pm} \mp V_{0}^{3}\right)^{2} G^{00}+4 g^{2} \phi^{2}\right] \\
& \quad \times \sqrt{-G} G^{m n} a_{1 n}^{ \pm}=0 .
\end{aligned}
$$

The explicit form of Eq. (65) in the background geometry (11) after the replacement $a_{1}^{a}(z)=z a_{1}^{\prime a}(z)$ will get the following form:

$$
\begin{aligned}
& \partial_{z}^{2} a_{1}^{\prime 0}+\frac{1}{z} \partial_{z} a_{1}^{0}+\left[\bar{\omega}_{0}^{2}-g^{2}\left(m_{q}+\sigma z^{2}\right)^{2}-\frac{1}{z^{2}}\right] a_{1}^{\prime 0}=0 \\
& \partial_{z}^{2} a_{1}^{\prime \pm}+\frac{1}{z} \partial_{z} a_{1}^{ \pm}+\left[\left(\bar{\omega}_{ \pm} \mp V_{0}^{3}\right)^{2}-g^{2}\left(m_{q}+\sigma z^{2}\right)^{2}-\frac{1}{z^{2}}\right] a_{1}^{\prime \pm} \\
& \quad=0 .
\end{aligned}
$$

Following earlier work $[47,48]$ we consider at first the case of ignoring the condensate term $4 g^{2} \phi^{2}$ in the equations of motion (66). In this case we have no shift of the squared mass and a solution to Eq. (66) for the $s$ th mode $a_{1 s}^{a}(z)$ in the Kaluza-Klein decomposition

$a_{1 n}^{a}=\sum_{s} f_{1 n}^{a s}\left(P^{0}\right) a_{1 s}^{a}\left(P^{0}, z\right)$

after applying UV boundary condition gets a form, which is the same as the one for the vector mesons:

$a_{1 s}^{a}(z)=c_{1} z J_{1}\left(\bar{m}_{a}^{s} z\right)$.

The mass spectra $\bar{m}_{a}^{s}$ of the $s$ states are

$\bar{m}_{0}^{s}=\bar{\omega}_{0}^{s}$,

$\bar{m}_{ \pm}^{s}=\left|\bar{\omega}_{ \pm}^{s} \mp V_{0}^{3}\right|$,

and $\bar{\omega}_{ \pm}^{s}=\bar{\omega}_{0}^{s}$ are the vacuum masses of the excited axialvector mesons. From (68) the mass splitting formula for the $a_{1}^{ \pm}$mesons will be written in the following form:

$\bar{m}_{ \pm}^{s}=\left|\bar{m}_{0}^{s} \mp V_{0}^{3}\right|$.
It is obvious that the mass spectrum formula (43) is available for the axial-vector field case as well. The distinction in $z_{I R}$ can be compensated by introducing extra boundary terms $S_{ \pm}$ similar to the ones for the vector meson case:

$S_{ \pm}= \pm\left. V_{0}^{3} \int \mathrm{d}^{4} x \sqrt{-G} G^{00} G^{m n} \bar{a}_{1 m}^{ \pm *} \bar{a}_{1 n}^{ \pm}\right|_{z=z_{I R}}$.

These boundary terms will shift back the value of the infrared boundary $z_{I R}^{ \pm}$to the same value $z_{I R}$ for all $a_{1}^{a}$ mesons. In the case, when $\phi^{2} \neq 0$, Eq. (60) can be solved in the UV $(z \rightarrow 0)$ and in the IR $\left(z \rightarrow z_{I R}\right)$ limits. In order to find the mass spectrum in this case it is reasonable to apply the IR boundary condition to the asymptotic solution found in the IR limit. For the IR asymptotic solution we shall take the $z \rightarrow z_{I R}$ limit from (60) and set $z=z_{I R}$ in the condensate term. Before performing this approximation let us compare numerically the last two terms in Eq. (60) when $z \rightarrow z_{I R}$. The approximate values are: $z_{I R}^{-1} \approx 0.33 \mathrm{GeV}, z_{I R} \approx 3(\mathrm{GeV})^{-1}$, $z_{I R}^{4} \approx 81(\mathrm{GeV})^{-4}, \sigma \approx(0.3)^{3}(\mathrm{GeV})^{3}, g^{2}=4 \pi^{2} / N_{c} \approx$ 13.2. Then $g^{2} \sigma^{2}\left(z_{I R}\right)^{4} \approx 0.06(\mathrm{GeV})^{2}$ and $1 /\left(z_{I R}\right)^{2}=0.1$ $(\mathrm{GeV})^{2}$. Thus, the $1 / z^{2}$ term contributes twice more than the $g^{2} \sigma^{2} z^{4}$ term and so we may make an approximation in (60) by setting $z=z_{I R}$ only in the condensate term and keeping the term $1 / z^{2}$ variable. In this limit the condensate term in the equations (60) becomes constant and the IR asymptotic solution of these equations is expressed in terms of the Bessel function $J_{1}$ :

$a_{1 s}^{a}=c z J_{1}\left(\bar{m}_{a}^{s} z\right)$.

Obviously, the UV boundary condition was applied to this solution. The mass spectrum $\bar{m}_{a}^{s}$ in (71) is expressed in terms of $z_{I R}$ :

$$
\begin{aligned}
\bar{m}_{0}^{s} & =\sqrt{\left(\bar{\omega}_{0}^{s}\right)^{2}-g^{2}\left[m_{q}+\sigma\left(z_{I R}\right)^{2}\right]^{2}} \\
& \approx \bar{\omega}_{0}^{s}-\frac{g^{2}\left[m_{q}+\sigma\left(z_{I R}\right)^{2}\right]^{2}}{2 \bar{\omega}_{0}^{s}} \\
\bar{m}_{ \pm}^{s} & =\sqrt{\left(\bar{\omega}_{ \pm}^{s} \mp V_{0}^{3}\right)^{2}-g^{2}\left[m_{q}+\sigma\left(z_{I R}\right)^{2}\right]^{2}} \\
& \approx\left|\bar{\omega}_{ \pm}^{s} \mp V_{0}^{3}\right|-\frac{g^{2}\left[m_{q}+\sigma\left(z_{I R}\right)^{2}\right]^{2}}{2\left|\bar{\omega}_{ \pm}^{s} \mp V_{0}^{3}\right|} .
\end{aligned}
$$

As is seen from (72) condensate term contributes to the mass of states with different $s$ differently. The mass splitting formula for the spectra (72) will be written as usual:

$$
\begin{aligned}
\bar{m}_{ \pm}^{s} \approx & \left|\bar{m}_{0}^{s} \mp V_{0}^{3}+\frac{g^{2}\left(m_{q}+\sigma\left(z_{I R}\right)^{2}\right)^{2}}{2 \bar{\omega}_{0}^{s}}\right| \\
& -\frac{g^{2}\left(m_{q}+\sigma\left(z_{I R}\right)^{2}\right)^{2}}{2\left|\bar{\omega}_{ \pm}^{s} \mp V_{0}^{3}\right|},
\end{aligned}
$$

and it has the splitting sign as in the case of absence of the condensate. In the case of taking into account the condensate term the mass spectrum formula (41) is valid for the mass $\bar{m}_{a}^{s}$ shifted by the condensate: 
$\bar{m}_{0}^{s} \simeq\left(s-\frac{1}{4}\right) \frac{\pi}{z_{I R}^{0}}$

$\bar{m}_{ \pm}^{s} \simeq\left(s-\frac{1}{4}\right) \frac{\pi}{z_{I R}^{ \pm}} \quad s=1,2,3 \ldots$

This time a shift in mass is not simply $\mp V_{0}^{3}$ as in the previous case and it has a contribution proportional to the condensate value $\phi\left(z_{I R}\right)$. This contribution depends on $s$, and therefore, $z_{I R}^{ \pm}$this time depends on $s$ as well. This complicates the introduction of the IR boundary terms compensating the $z_{I R}^{ \pm}$ distinguished with the $z_{I R}^{0}$, which would be unique for all $s$ states. For this case we have to introduce the boundary term for each state $s$, which depends on the mass $\bar{\omega}_{ \pm}^{s}$ of this state as well,

$$
\begin{aligned}
S_{ \pm}^{s}= & \left( \pm V_{0}^{3}+\frac{g^{2}\left(m_{q}+\sigma\left(z_{I R}\right)^{2}\right)^{2}}{2\left|\bar{\omega}_{ \pm}^{s} \mp V_{0}^{3}\right|}\right) \\
& \times\left.\int \mathrm{d}^{4} x \sqrt{-G} G^{00} G^{m n} f_{1 m}^{ \pm s *} f_{1 n}^{ \pm s}\left(a_{1 s}^{ \pm}\right)^{2}\right|_{z=z_{I R}},
\end{aligned}
$$

and the total boundary term will be a sum of boundary terms of the $s$ modes,

$S_{ \pm}=\sum_{s} S_{ \pm}^{s}$

We should compensate as well the shift in the mass spectrum of the neutral $a_{1}^{0}$ meson, which was caused by the condensate term. As is seen from Eq. (72) the condensate shift in the $\bar{m}_{0}^{s}$ spectrum is different for the different $s$ states. This means one should distinguish values of $z_{I R}$, these being $s$ dependent. In order to avoid the distinguished $z_{I R}$ we introduce an additional boundary term for the each Kaluza-Klein $s$ mode and sum these terms:

$$
\begin{aligned}
S_{0}= & \frac{1}{2} g^{2}\left(m_{q}+\sigma\left(z_{I R}\right)^{2}\right)^{2} \\
& \times\left.\int \mathrm{d}^{4} x \sqrt{-G} G^{00} G^{m n} \sum_{s} \frac{1}{\bar{\omega}_{0}^{s}} f_{1 m}^{0 s *} f_{1 n}^{0 s}\left(a_{1 s}^{0}\right)^{2}\right|_{z=z_{I R}} .
\end{aligned}
$$

One should notice that in [52], where the axial-vector meson's mass spectrum including a condensate contribution was obtained in the vacuum case, an approximation was performed which shifts the boundary condition at $z_{I R}$ and, thus, the condensate contribution to the mass of meson was taken into account. To do a shift in such a way is equivalent to introducing the boundary term $S_{0}$ in our case. ${ }^{2}$

\footnotetext{
${ }^{2}$ Although the condensate shift in the squared mass spectrum is the same for all Kaluza-Klein states $s$ the approximation here takes it into account the mass spectrum depending on the mass $\omega^{s}$ of the state. In the approximation made in [52] the shift in the IR boundary condition is the same for all $s$ states.
}

\section{$4.2 \pi$ Mesons in the isospin medium}

Following [47], we introduce the charged $\pi^{a}$ and $\chi^{a}$ fields by means of the $\pi^{i}$ and $\chi^{i}$ fields, respectively, as below:

$\pi_{m}^{0}=\pi_{m}^{3}, \quad \chi_{m}^{0}=\chi_{m}^{3}$,

$\pi_{m}^{ \pm}=\frac{1}{\sqrt{2}}\left(\pi_{m}^{1} \pm i \pi_{m}^{2}\right), \quad \chi_{m}^{ \pm}=\frac{1}{\sqrt{2}}\left(\chi_{m}^{1} \pm i \chi_{m}^{2}\right)$.

In the AdS/CFT correspondence the UV boundary values of the $\pi^{a}$ fields ( $a=0, \pm$ ) will be mapped to the pion fields of the dual QCD. The Fourier components for the $\pi^{a}$ and $\chi^{a}$ fields are written as

$$
\begin{aligned}
& \pi_{m}^{0}(t, z)=\int \frac{\mathrm{d} \widetilde{\omega}_{0}}{2 \pi} e^{-i \widetilde{\omega}_{0} t} \pi_{m}^{0}\left(\widetilde{\omega}_{0}, z\right), \\
& \chi_{m}^{0}(t, z)=\int \frac{\mathrm{d} \widetilde{\omega}_{0}}{2 \chi} e^{-i \widetilde{\omega}_{0} t} \chi_{m}^{0}\left(\widetilde{\omega}_{0}, z\right), \\
& \pi_{m}^{ \pm}(t, z)=\int \frac{\mathrm{d} \widetilde{\omega}_{ \pm}}{2 \pi} e^{-i \widetilde{\omega}_{ \pm} t} \pi_{m}^{ \pm}\left(\widetilde{\omega}_{ \pm}, z\right), \\
& \chi_{m}^{ \pm}(t, z)=\int \frac{\mathrm{d} \widetilde{\omega}_{ \pm}}{2 \pi} e^{-i \widetilde{\omega}_{ \pm} t} \chi_{m}^{ \pm}\left(\widetilde{\omega}_{ \pm}, z\right),
\end{aligned}
$$

where $\widetilde{\omega}_{a}$ are the masses of the $\pi^{a}$ (and $\chi^{a}$ ) fields in the absence isospin background $V_{0}^{3}$, i.e. they are the vacuum masses and do not include the contribution of the condensate. As in the $\rho^{a}$ and $a_{1}^{a}$ meson cases the vacuum masses $\widetilde{\omega}_{a}$ of the $\pi^{a}$ fields do not depend on $\mu_{P}$ and $\mu_{N}$ and their equality $\widetilde{\omega}_{ \pm}=\widetilde{\omega}_{0}$ is right in the isospin medium case as well. The action (57) in terms of the $\pi^{a}$ and $\chi^{a}$ fields splits into three independent parts:

$S_{\pi+\chi}=\int \mathrm{d}^{4} x \int_{0}^{z_{I R}} \mathrm{~d} z \sqrt{-G}\left\{\widetilde{\mathcal{L}}^{(0)}+\widetilde{\mathcal{L}}^{(+)}+\widetilde{\mathcal{L}}^{(-)}\right\}$,

where

$$
\begin{aligned}
\widetilde{\mathcal{L}}^{(0)}= & \frac{1}{4 g^{2}} G^{z z} G^{00} \omega_{0}^{2}\left(\partial_{z} \chi^{0}\right)^{2}+\phi^{2} G^{z z}\left(\partial_{z} \pi^{0}\right)^{2} \\
& +\phi^{2} \omega_{0}^{2} G^{00}\left(\chi^{0}-\pi^{0}\right)^{2}, \\
\widetilde{\mathcal{L}}^{( \pm)}= & \frac{1}{4 g^{2}} G^{z z} G^{00} \omega_{ \pm}^{2}\left(\partial_{z} \chi^{ \pm}\right)^{2}+\phi^{2} G^{00} \omega_{ \pm}^{2}\left(\chi^{ \pm}\right)^{2} \\
& +\phi^{2} G^{z z}\left(\partial_{z} \pi^{ \pm}\right)^{2} \\
& +\phi^{2} G^{00} \pi^{ \pm}\left(\omega_{ \pm} \mp V_{0}^{3}\right)\left[\pi^{ \pm}\left(\omega_{ \pm} \mp V_{0}^{3}\right)-2 \chi^{ \pm} \omega_{ \pm}\right] .
\end{aligned}
$$

The equations of motion for these scalar fields are obtained in [47] from the action (79) and are summarized as follows:

$$
\begin{aligned}
& \chi^{0}-\pi^{0}=\frac{1}{4 g^{2} \phi^{2} \sqrt{-G} G^{00}} \partial_{z}\left(\sqrt{-G} G^{z z} G^{00} \partial_{z} \chi^{0}\right), \\
& \omega_{0}^{2}\left(\chi^{0}-\pi^{0}\right)=-\frac{1}{\phi^{2} \sqrt{-G} G^{00}} \partial_{z}\left(\phi^{2} \sqrt{-G} G^{z z} \partial_{z} \pi^{0}\right),
\end{aligned}
$$


and

$$
\begin{aligned}
& \omega_{ \pm} \chi^{ \pm}-\left(\omega_{ \pm} \mp V_{0}^{3}\right) \pi^{ \pm}=\frac{\omega_{ \pm}}{4 g^{2} \phi^{2} \sqrt{-G} G^{00}} \\
& \quad \times \partial_{z}\left(\sqrt{-G} G^{z z} G^{00} \partial_{z} \chi^{ \pm}\right), \\
& \left(\omega_{ \pm} \mp V_{0}^{3}\right)\left[\omega_{ \pm} \chi^{ \pm}-\left(\omega_{ \pm} \mp V_{0}^{3}\right) \pi^{ \pm}\right] \\
& =-\frac{1}{\phi^{2} \sqrt{-G} G^{00}} \partial_{z}\left(\phi^{2} \sqrt{-G} G^{z z} \partial_{z} \pi^{ \pm}\right) .
\end{aligned}
$$

The boundary terms arising on obtaining these equations are

$$
\left.\int \mathrm{d}^{4} p \phi^{2} \sqrt{-G} G^{z z} \pi^{a *}(z) \partial_{z} \pi^{a}(z)\right|_{z_{I R}} ^{z_{U V}}=0,
$$

and the boundary conditions imposed on the $\pi^{a}$ fields are the usual ones: $\left.\partial_{z} \pi^{a}(z)\right|_{z_{I R}}=0$ and $\left.\pi^{a}(z)\right|_{z_{U V}}=0$. Comparing the first equations with the second ones in (81) and in (82) one can establish the following relations between the $\partial_{z}$ derivatives of the $\pi$ and $\chi$ fields:

$\widetilde{\omega}_{0}^{2} G^{00} \partial_{z} \chi^{0}=4 g^{2} \phi^{2} \partial_{z} \pi^{0}$,

$\widetilde{\omega}_{ \pm}\left(\widetilde{\omega}_{ \pm} \mp V_{0}^{3}\right) G^{00} \partial_{z} \chi^{ \pm}=4 g^{2} \phi^{2} \partial_{z} \pi^{ \pm}$.

Taking the derivative from Eqs. (81) and (82) and taking into account the relations (84), the equation of motion for the $\pi^{a}$ field will be separated from the one for the $\chi^{a}$ field:

$$
\begin{aligned}
\partial_{z} & {\left[\frac{1}{\phi^{2} \sqrt{-G} G^{00}} \partial_{z}\left(\phi^{2} \sqrt{-G} G^{z z} \partial_{z} \pi^{0}\right)\right] } \\
& -\left(\widetilde{\omega}_{0}^{2}+4 g^{2} \phi^{2} G_{00}\right) \partial_{z} \pi^{0}=0, \\
\partial_{z} & {\left[\frac{1}{\phi^{2} \sqrt{-G} G^{00}} \partial_{z}\left(\phi^{2} \sqrt{-G} G^{z z} \partial_{z} \pi^{ \pm}\right)\right] } \\
- & {\left[\left(\widetilde{\omega}_{ \pm} \mp V_{0}^{3}\right)^{2}+4 g^{2} \phi^{2} G_{00}\right] \partial_{z} \pi^{ \pm}=0 . }
\end{aligned}
$$

The physical situation with the $\pi$ fields is more complicated in comparison with the vector and axial-vector fields considered above. As is seen from the action (57) the $\pi$ fields interact with three other fields: the background condensate field $\phi$, the longitudinal axial-vector field $\chi$, and the background isospin field $V_{0}^{3}$. In the action (57) the interaction with the $\phi$ field gives a contribution to the mass term of the $\pi$ field and this contribution is the same for all $\pi^{a}$ components. The interaction with the isospin field $V_{0}^{3}$ gives different contributions to the mass terms of the different $\pi^{a}$ components in the equations of motion and, thus, splits the mass of these fields, which can be inferred from Eq. (85). The interaction with the $\chi$ field lifts up the order in the equations of motion, which can be seen on taking the $\chi$ field to be zero in the action (57) or in Eqs. (81) and (82). In the case $\chi=0$ we have second order differential equations, instead of the third order one in Eq. (85). Another observation is that the $\phi(z)$ field modifies the AdS metric (11). It is easy to see this in the $\chi=0$ case of the equations for the $\pi$ fields in (81) and (82). Choosing the following notation in the metric tensor:
$G_{M M}=\phi^{-4 / 3}(z) \mathbf{G}_{M M}, \quad G^{M M}=\phi^{4 / 3}(z) \mathbf{G}^{M M}$,

we get the modified background geometry:

$\mathrm{d} s^{2}=\frac{R^{2}}{z^{2} \phi^{4 / 3}}\left(-\mathrm{d} t^{2}+\mathrm{d} z^{2}+\mathrm{d} \vec{x}^{2}\right)$.

Then

$\phi^{2} \sqrt{-G} G^{z z}=\sqrt{-\mathbf{G}} \mathbf{G}^{z z}, \quad \phi^{2} \sqrt{-G} G^{00}=\sqrt{-\mathbf{G}} \mathbf{G}^{00}$,

and the equations for the $\pi$ fields in (81) and (82) get the form

$$
\begin{aligned}
& -\omega_{0}^{2} \sqrt{-\mathbf{G}} \mathbf{G}^{00} \pi^{0}+\partial_{z}\left(\sqrt{-\mathbf{G}} \mathbf{G}^{z z} \partial_{z} \pi^{0}\right)=0, \\
& -\sqrt{-\mathbf{G}} \mathbf{G}^{00}\left(\omega_{ \pm} \mp V_{0}^{3}\right)^{2} \pi^{ \pm}+\partial_{z}\left(\sqrt{-\mathbf{G}} \mathbf{G}^{z z} \partial_{z} \pi^{ \pm}\right)=0 .
\end{aligned}
$$

As is seen from (89) ignorance of the $\chi$ field in the action leads to the absence of the condensate term $4 g^{2} \phi^{2}$ in the equation of motion. This case is useful for a comparison with the mass splitting formula for pions obtained in $[34,47,48]$. Equation (89) is, in fact, the five-dimensional Klein-Gordon equation for the free $\pi^{a}$ field with zero five-dimensional mass term in the background geometry (87):

$$
\begin{aligned}
& \frac{1}{\sqrt{-\mathbf{G}}} \partial_{z}\left(\sqrt{-\mathbf{G}} \mathbf{G}^{z z} \partial_{z} \pi^{0}\right) \\
& \quad+\frac{1}{\sqrt{-\mathbf{G}}} D_{\mu}^{(a)}\left(\sqrt{-\mathbf{G}} \mathbf{G}^{\mu \mu^{\prime}} D_{\mu^{\prime}}^{(a)} \pi^{a}\right) \\
& =\frac{1}{\sqrt{-\mathbf{G}}} D_{M}^{(a)}\left(\sqrt{-\mathbf{G}} \mathbf{G}^{M M^{\prime}} D_{M^{\prime}}^{(a)} \pi^{a}\right)=0 .
\end{aligned}
$$

Consequently, the equation of motion for the $\pi^{a}$ field interacting with the $\phi$ field in the background geometry (11) is equivalent to the one for the free $\pi^{a}$ field in the modified geometry (87). It is obvious that this equivalence does not depend on the isospin medium and is valid for the $V_{0}^{3}=0$ case as well. It should be noticed that the background geometry modification by the scalar field $\phi$ was observed in $[56,57]$ also. Using (90) we can derive a formula determining the effective mass of the $\pi$ fields. In order to make the correspondence similar to previous sections let us write the KleinGordon equation in a four-dimensional curved space-time with the new metric $\mathbf{G}$ for the massive scalar field $\pi^{a}$ interacting with the constant external gauge field $V_{0}^{3}$ :

$\frac{1}{\sqrt{-\mathbf{G}}} D_{\mu}^{(a)}\left(\sqrt{-\mathbf{G}} \mathbf{G}^{\mu \mu^{\prime}} D_{\mu^{\prime}}^{(a)} \pi^{a}\right)-\left(m^{a}\right)^{2} \pi^{a}=0$,

where $m^{a}$ is the mass of the $\pi^{a}$ field. If we are to consider Eq. (91) as the UV limit of Eq. (90), then the eigenvalues of the operator $-\frac{1}{\sqrt{-\mathbf{G}}} \partial_{z}\left(\sqrt{-\mathbf{G}} \mathbf{G}^{z z^{\prime}} \partial_{z^{\prime}}\right)$ in (90) will correspond to the squared mass $\left(m^{a}\right)^{2}$ of the $4 \mathrm{D}$ vector field $\pi^{a}$ in (91). So, we may admit an equality of the eigenvalues of 
this operator to the effective mass of the $\pi$ field defined on this boundary:

$$
-\frac{1}{\sqrt{-\mathbf{G}}} \partial_{z}\left(\sqrt{-\mathbf{G}} \mathbf{G}^{z z^{\prime}} \partial_{z^{\prime}} \pi^{a}\right)=\left(m^{a}\right)^{2} \pi^{a} .
$$

Equation (92) is the one determining the effective mass of the $\pi$ field in the isospin medium by means of the eigenvalue of the derivative operator over the extra dimension. For the Kaluza-Klein mode $\pi_{s}^{a}$ in the decomposition

$\pi^{a}(x, z)=\sum_{s} b^{a s}\left(P^{0}\right) \pi_{s}^{a}\left(P^{0}, z\right)$

a solution to Eq. (83) is expressed in terms of the Bessel functions $J_{1}$ and $Y_{1}$ :

$\pi_{s}^{a}(z)=\frac{z}{\tilde{\mathbf{m}}_{a}^{s}}\left[c_{1} J_{1}\left(\tilde{\mathbf{m}}_{a}^{s} z\right)+c_{2} Y_{1}\left(\tilde{\mathbf{m}}_{a}^{s} z\right)\right]$.

Here the eigenvalues $\tilde{\mathbf{m}}_{a}^{s}$ are the mass spectrum:

$\tilde{\mathbf{m}}_{0}^{s}=\widetilde{\omega}_{0}^{s}$,

$\tilde{\mathbf{m}}_{ \pm}^{s}=\left|\widetilde{\omega}_{ \pm}^{s} \mp V_{0}^{3}\right|=\left|\tilde{\mathbf{m}}_{0}^{s} \mp V_{0}^{3}\right|$,

and it has a splitting sign opposite to the one in $[34,47,48]$. For the solution (93) the spectrum (41) is available due to the Neumann boundary condition at the IR boundary and, obviously, in this case it can be introduced usual additional boundary terms $S_{ \pm}$in order to avoid the $z_{I R}^{ \pm}$distinguished from the $z_{I R}^{0}$ introduced in previous sections:

$$
\begin{aligned}
S_{ \pm} & = \pm V_{0}^{3} \int \mathrm{d}^{4} x \phi^{2} \sqrt{-G} G^{z z} G^{m n}\left(\left(\pi_{m}^{ \pm}\right)^{*} \pi_{n}^{ \pm}\right)_{z=z_{I R}} \\
& = \pm V_{0}^{3} \int \mathrm{d}^{4} x \sqrt{-\mathbf{G}} \mathbf{G}^{z z}\left(\left(\pi^{ \pm m}\right)^{*} \pi_{m}^{ \pm}\right)_{z=z_{I R}} .
\end{aligned}
$$

It is interesting to consider the $\chi \neq 0$ case as well. In this case Eq. (85) contains the condensate term $4 g^{2} \phi^{2}$ and can be solved in the UV and IR limits of $z$. As is well known, in the free case the equation of motion for the $\pi^{a}$ field was solved either in the chiral limit $\left(m_{q}=0\right)$ [58,59] or in the zero condensate limit $(\phi=0)$. In our isospin medium case Eq. (85) will be solved in the UV and IR limits of $z$, i.e. in the $z \rightarrow 0$ and $z \rightarrow z_{I R}$ limits. In order to find the UV limit solution we take the $z \rightarrow 0$ limit from Eq. (85), which leads to the usual Bessel equation for the $\partial_{z} \pi^{a}(z)$ :

$$
\begin{aligned}
& \partial_{z}^{2}\left(\partial_{z} \pi^{0}\right)-\frac{1}{z} \partial_{z}\left(\partial_{z} \pi^{0}\right)+\left[\widetilde{\omega}_{0}^{2}-g^{2} m_{q}^{2}+\frac{1}{z^{2}}\right]\left(\partial_{z} \pi^{0}\right)=0 \\
& \partial_{z}^{2}\left(\partial_{z} \pi^{ \pm}\right)-\frac{1}{z} \partial_{z}\left(\partial_{z} \pi^{ \pm}\right) \\
& \quad+\left[\left(\widetilde{\omega}_{ \pm} \mp V_{0}^{3}\right)^{2}-g^{2} m_{q}^{2}+\frac{1}{z^{2}}\right]\left(\partial_{z} \pi^{ \pm}\right)=0 .
\end{aligned}
$$

Denoting $\partial_{z} \pi^{a}={ }_{z} P^{a}(z)$ we shall get the Bessel equation for $P^{a}(z)$, which has a solution expressed in terms of the Bessel functions $J_{0}$ and $Y_{0}$ for the Kaluza-Klein mode $\pi_{s}^{a}$ :

$P_{s}^{a}(z)=c_{1} J_{0}\left(\tilde{\mathbf{m}}_{a}^{s} z\right)+c_{2} Y_{0}\left(\tilde{\mathbf{m}}_{a}^{s} z\right)$.
Here $\tilde{\mathbf{m}}_{a}^{s}$ denotes the square root of the constant in the last terms in Eq. (91):

$\tilde{\mathbf{m}}_{0}^{s}=\sqrt{\left(\widetilde{\omega}_{0}^{s}\right)^{2}-g^{2} m_{q}^{2}}$,

$\tilde{\mathbf{m}}_{ \pm}^{s}=\sqrt{\left(\widetilde{\omega}_{ \pm}^{s} \mp V_{0}^{3}\right)^{2}-g^{2} m_{q}^{2}}$

Using the differentiation formula for the Bessel functions

$\frac{\mathrm{d}}{\mathrm{d} x}\left[x^{\nu} Z_{v}(x)\right]=x^{v} Z_{v-1}(x)$,

we find the solution $\pi_{s}^{a}$ in terms of the Bessel functions $J_{1}$ and $Y_{1}$,

$\pi_{s}^{a}(z)=\frac{z}{\tilde{\mathbf{m}}_{a}^{s}}\left[c_{1} J_{1}\left(\tilde{\mathbf{m}}_{a}^{s} z\right)+c_{2} Y_{1}\left(\tilde{\mathbf{m}}_{a}^{s} z\right)\right]$.

The boundary conditions which were imposed on the solution $\pi_{s}^{a}$ when deriving (85) are the usual ones: $\left.\partial_{z} \pi_{s}^{a}\right|_{z_{I R}}=0$ and $\left.\pi_{s}^{a}\right|_{0}=0$. Similarly to the previous section, the application of the UV boundary condition on (100) gives $c_{2}=0$.

Now we solve Eq. (85) in the $z \rightarrow z_{I R}$ limit. We ignore $m_{q}$ in the condensate term due to $m_{q} \ll \sigma z_{I R}^{2}$. Making the substitution $\partial_{z} \pi^{a}=\frac{1}{z} P^{\prime a}(z)$ in these equations we obtain the following explicit form of them:

$$
\begin{aligned}
& \partial_{z}^{2} P^{\prime 0}(z)+\frac{1}{z} \partial_{z} P^{\prime 0}(z)+\left[\widetilde{\omega}_{0}^{2}-g^{2} \sigma^{2} z^{4}-\frac{4}{z^{2}}\right] P^{\prime 0}(z)=0 \\
& \partial_{z}^{2} P^{\prime \pm}(z)+\frac{1}{z} \partial_{z} P^{\prime \pm}(z) \\
& \quad+\left[\left(\widetilde{\omega}_{ \pm} \mp V_{0}^{3}\right)^{2}-g^{2} \sigma^{2} z^{4}-\frac{4}{z^{2}}\right] P^{\prime \pm}(z)=0
\end{aligned}
$$

In order to get the IR asymptotic solution we shall take the $z \rightarrow z_{I R}$ limit from the equations in (101) and set $z=z_{I R}$ in the condensate term. A numerical comparison of terms $g^{2} \sigma^{2} z^{4}$ and $1 / z^{2}$ done when solving the Eq. (66) is available for Eq. (101) as well. As a result of this approximation we shall find the following Bessel function asymptotic solution for the equations in (101):

$P_{s}^{\prime a}(z)=c_{1}^{\prime} J_{2}\left(\tilde{\mathbf{M}}_{a}^{s} z\right)+c_{2}^{\prime} Y_{2}\left(\tilde{\mathbf{M}}_{a}^{s} z\right)$,

where $\tilde{\mathbf{M}}_{a}^{s}$ denotes the square root of the value of brackets without the $4 / z^{2}$ term in (102) at $z_{I R}$ :

$$
\begin{aligned}
\tilde{\mathbf{M}}_{0}^{s} & =\sqrt{\left(\widetilde{\omega}_{0}^{s}\right)^{2}-g^{2} \sigma^{2} z_{I R}^{4}} \approx \widetilde{\omega}_{0}^{s}-\frac{g^{2} \sigma^{2} z_{I R}^{4}}{2 \widetilde{\omega}_{0}^{s}}, \\
\tilde{\mathbf{M}}_{ \pm}^{s} & =\sqrt{\left(\widetilde{\omega}_{ \pm}^{s} \mp V_{0}^{3}\right)^{2}-g^{2} \sigma^{2} z_{I R}^{4}} \\
& \approx\left|\widetilde{\omega}_{ \pm}^{s} \mp V_{0}^{3}\right|-\frac{g^{2} \sigma^{2} z_{I R}^{4}}{2\left|\widetilde{\omega}_{ \pm}^{s} \mp V_{0}^{3}\right|} .
\end{aligned}
$$

From (103) we can write a mass splitting formula for this case:

$\tilde{\mathbf{M}}_{ \pm}^{s} \approx\left|\tilde{\mathbf{M}}_{0}^{s} \mp V_{0}^{3}+\frac{g^{2} \sigma^{2} z_{I R}^{4}}{2 \widetilde{\omega}_{0}^{s}}\right|-\frac{g^{2} \sigma^{2} z_{I R}^{4}}{2\left|\widetilde{\omega}_{0}^{s} \mp V_{0}^{3}\right|}$. 
It is obvious all three $\tilde{\mathbf{M}}_{a}^{s}$ get the same value in the $V_{0}^{3} \rightarrow 0$ limit.

Using (98) and the property $Z_{-n}=(-1)^{n} Z_{n}$ of the Bessel functions we can write the solutions $\pi_{s}^{a}$ :

$\pi_{s}^{a}(z)=-\frac{1}{z}\left[c_{1}^{\prime} J_{1}\left(\tilde{\mathbf{M}}_{a}^{s} z\right)+c_{2}^{\prime} Y_{1}\left(\tilde{\mathbf{M}}_{a}^{s} z\right)\right]$.

The UV boundary condition on this solution leads to $c_{2}=0$. In the UV limit, when $z \rightarrow 0$, the $\sigma^{2} z^{4}$ term drops out and the solution to (85) will be the same as the one for Eq. (66). The general solution to Eq. (85) will be a linear combination of these asymptotic solutions. It can be shown that the constants $c_{2}$ and $c_{2}^{\prime}$ are zero in this case as well and the general solution has got the form

$\pi_{s}^{a}(z)=c_{1} z J_{1}\left(\tilde{\mathbf{m}}_{a}^{s} z\right)+\frac{c_{1}^{\prime}}{z} J_{1}\left(\tilde{\mathbf{M}}_{a}^{s} z\right)$.

We shall apply the Neumann boundary condition at the IR boundary only on the IR asymptotic part solution (106), i.e. only on the second term in (106). This gives us a discrete mass spectrum in terms of the zeros $\alpha_{2}^{s}$ of the Bessel function $J_{2}$ :

$\tilde{\mathbf{M}}_{0}^{s}=\frac{\alpha_{2}^{s}}{\tilde{z}_{I R}^{0}}$,

$\tilde{\mathbf{M}}_{ \pm}^{s}=\frac{\alpha_{2}^{s}}{\tilde{z}_{I R}^{ \pm}} ; \quad s=1,2,3 \ldots$

In order to verify our consideration of $\tilde{\mathbf{M}}_{a}^{s}$ as an effective mass we should reduce Eqs. (85) to the form in Eq. (89) in the UV limit $z \rightarrow 0$. Let us write Eq. (85) in terms of the induced metric (86):

$$
\begin{aligned}
\partial_{z}[ & \frac{1}{\sqrt{-\mathbf{G}} \mathbf{G}^{00}} \partial_{z}\left(\sqrt{-\mathbf{G}} \mathbf{G}^{z z} \partial_{z} \pi^{a}\right) \\
& \left.+\left(D_{0}^{a} D_{0}^{a}+4 g^{2} \phi^{5 / 2} \mathbf{G}_{00}\right) \pi^{a}\right] \\
= & 4 g^{2} \pi^{a} \partial_{z}\left(\phi^{5 / 2} \mathbf{G}_{00}\right) .
\end{aligned}
$$

The right hand side of this equation in the UV limit gives zero due to the UV boundary condition $\left.\pi^{a}\right|_{0}=0$. Then the free constant obtained in the left hand side can be chosen zero and (107) will be written as

$$
\begin{aligned}
& \frac{1}{\sqrt{-\mathbf{G}}} \partial_{z}\left(\sqrt{-\mathbf{G}} \mathbf{G}^{z z} \partial_{z} \pi^{a}\right) \\
& +\mathbf{G}^{00}\left(D_{0}^{a} D_{0}^{a}+4 g^{2} \phi^{5 / 2} \mathbf{G}_{00}\right) \pi^{a}=0 .
\end{aligned}
$$

This equation has the form of the equations in (89) and it can be considered as a Klein-Gordon equation in the background geometry with the metric $\mathbf{G}_{M N}$. Then the second term will be considered as an effective mass including the condensate contribution. This proves that the derivative operator over the extra dimension $z$ determines the effective mass through the eigenvalues $\tilde{\mathbf{M}}_{a}^{s}$ in this non-zero condensate term case as well.
In this case the additional boundary terms compensating for the distinguished $z_{I R}$ will be dependent on the condensate $g^{2} \sigma^{2} z_{I R}^{4}$ and the mass of the state $\widetilde{\omega}_{a}^{s}$ :

$$
\begin{aligned}
S_{ \pm}= & \sum_{s}\left( \pm V_{0}^{3}+\frac{g^{2} \sigma^{2} z_{I R}^{4}}{2\left|\widetilde{\omega}_{0}^{s} \mp V_{0}^{3}\right|}\right) \\
& \times \int \mathrm{d}^{4} x \phi^{2} \sqrt{-G} G^{z z}\left(b^{ \pm s}\right)^{2}\left(\pi_{s}^{ \pm}\right)_{z=z_{I R}}^{2} \\
= & \sum_{s}\left( \pm V_{0}^{3}+\frac{g^{2} \sigma^{2} z_{I R}^{4}}{2\left|\widetilde{\omega}_{0}^{s} \mp V_{0}^{3}\right|}\right) \\
& \times \int \mathrm{d}^{4} x \sqrt{-\mathbf{G}} \mathbf{G}^{z z}\left(b^{ \pm s}\right)^{2}\left(\pi_{s}^{ \pm}\right)_{z=z_{I R}}^{2}, \\
S_{0}= & \sum_{s} \frac{g^{2} \sigma^{2} z_{I R}^{4}}{2 \widetilde{\omega}_{0}^{s}} \int \mathrm{d}^{4} x \sqrt{-\mathbf{G}} \mathbf{G}^{z z}\left(b^{0 s}\right)^{2}\left(\pi_{s}^{0}\right)_{z=z_{I R}}^{2} .
\end{aligned}
$$

Since the condensate term $4 g^{2} \phi^{2}$ shifts the masses of all pions, the boundary terms above include it also.

\section{Discussion}

Here we obtain a formula for the meson's effective mass in the isospin medium using holography. We observe that interaction with the isospin background leads to the necessity of distinguishing of the infrared boundaries for different mesons from the same isotriplet. To bring all infrared boundaries to the same value requires one to introduce additional boundary terms, which leads to a modification of the boundary condition in the presence of an isospin background field. Indeed, the modification of the boundary condition at the infrared boundary is known from Refs. [37,52]. In [52], the axial-vector field interacting with the condensate field gets a contribution in mass and this results in a modification in the boundary condition at $z_{I R}$. The shift in the boundary condition which was made in this work is equivalent to adding a new boundary term proportional to the condensate field. Here we have an isospin field interaction with the $\rho$ mesons, which contributes to the mass differently for the $\rho^{+}$and $\rho^{-}$mesons. This results in the introduction of different boundary terms for these mesons. In the case of $a_{1}$ and $\pi$ mesons we have two fields (condensate field and isospin field), the interaction with which contributes to the mass of these mesons. In the result we have a double shift in the mass and this requires one to introduce boundary terms proportional to both these fields.

Acknowledgments The author thanks Joshua Erlich for useful discussion of adding new boundary terms and for commenting on the manuscript. He acknowledges Chanyong Park and Bum-Hoon Lee for useful discussions at the beginning stage of this work. The author thanks TUBITAK organization of Turkey for the grant 2221 - Fellowships for Visiting Scientists and Scientists on Sabbatical Leave and Physics Department of Gazi University, where the main part of present work was done, for the hospitality during visiting. 
Open Access This article is distributed under the terms of the Creative Commons Attribution 4.0 International License (http://creativecomm ons.org/licenses/by/4.0/), which permits unrestricted use, distribution, and reproduction in any medium, provided you give appropriate credit to the original author(s) and the source, provide a link to the Creative Commons license, and indicate if changes were made. Funded by SCOAP ${ }^{3}$.

\section{References}

1. D.T. Son, M.A. Stephanov, Phys. Rev. Lett. 86, 592 (2001). arXiv:hep-ph/0005225

2. J.M. Maldacena, Adv. Theor. Math. Phys. 2, 231 (1998)

3. J.M. Maldacena, Int. J. Theor. Phys. 38, 1113 (1999). arXiv:hep-th/9711200

4. S.S. Gubser, I.R. Klebanov, A.M. Polyakov, Phys. Lett. B 428, 105 (1998). arXiv:hep-th/9802109

5. E. Witten, Adv. Theor. Math. Phys. 2, 253 (1998). arXiv:hep-th/9802150

6. O. Aharony, S.S. Gubser, J.M. Maldacena, H. Ooguri, Y. Oz, Phys. Rep. 323, 183 (2000). arXiv:hep-th/9905111

7. H. Boschi-Filho, N.R.F. Braga, JHEP 0305, 009 (2003). arXiv:hep-th/0212207

8. H. Boschi-Filho, N.R.F. Braga, Eur. Phys. J. C 32, 529 (2004). arXiv:hep-th/0209080

9. J. Erlich, E. Katz, D.T. Son, M.A. Stephanov, Phys. Rev. Lett. 95, 261602 (2005). arXiv:hep-ph/0501128

10. A. Karch, E. Katz, D.T. Son, M.A. Stephanov, Phys. Rev. D 74, 015005 (2006). arXiv:hep-ph/0602229

11. L. Da Rold, A. Pomarol, Nucl. Phys. B 721, 79 (2005). arXiv:hep-ph/0501218

12. L. Da Rold, A. Pomarol, JHEP 0601, 157 (2006). arXiv:hep-ph/0510268

13. S.J. Brodsky, G.F. de Teramond, H.G. Dosch, J. Erlich, Phys. Rept. 584, 1 (2015). arXiv:1407.8131 [hep-ph]

14. J. Erlich, Contemp. Phys. 56, 159 (2015). arXiv:1407.5002 [hep$\mathrm{ph}]$

15. D.K. Hong, T. Inami, H.-U. Yee, Phys. Lett. B 646, 165 (2007). arXiv:hep-ph/0609270

16. Z. Abidin, C. Carlson, Phys. Rev. D 79, 115003 (2009). arXiv:0903.4818 [hep-ph]

17. E. Witten, Adv. Theor. Math. Phys. 2, 505 (1998). arXiv:hep-th/9803131

18. K. Ghoroku, M. Yahiro, Phys. Rev. D 73, 125010 (2006). arXiv:hep-ph/0512289

19. P. Colangelo, F. Giannuzzi, S. Nicotri, V. Tangorra, Eur. Phys. J. C 72, 2096 (2012). arXiv:1112.4402 [hep-ph]

20. P. Colangelo, F. Giannuzzi, S. Nicotri, JHEP 1205, 076 (2012). arXiv:1201.1564 [hep-ph]

21. M. Fujita, K. Fukushima, T. Misumi, M. Murata, Phys. Rev. D 80, 035001 (2009). arXiv:0903.2316 [hep-ph]

22. A.S. Miranda, C.A. Ballon Bayona, H. Boschi-Filho, N.R.F. Braga, JHEP 0911, 119 (2009). arXiv:0909.1790 [hep-th]

23. L.A.H. Mamani, A.S. Miranda, H. Boschi-Filho, N.R.F. Braga, JHEP 1403, 058 (2014). arXiv:1312.3815 [hep-th]

24. A.S. Miranda, C.A. Ballon Bayona, H. Boschi-Filho, N.R.F. Braga, Nucl. Phys. Proc. Suppl. 199, 107-112 (2010). arXiv:0910.4319 [hep-th]

25. Z. Li, B.-Q. Ma, Phys. Rev. D 89, 015014 (2014). arXiv:1312.3451 [hep-ph]

26. C. Park, Phys. Rev. D 81, 045009 (2010). arXiv:0907.0064 [hep$\mathrm{ph}]$
27. J. Casalderrey-Solana, H. Liu, D. Mateos, K. Rajagopal, U.A. Wiedemann, Gauge/string duality, hot QCD and heavy ion collisions (Cambridge University Press, Cambridge, 2014)

28. E. Papantonopoulos, From Gravity to Thermal Gauge Theories: The AdS/CFT Correspondence. Lecture Notes in Physics, vol. 828 (Springer, Berlin, 2011)

29. I. Ya, Aref'eva. Phys. Usp. 57, 527 (2014)

30. A. Parnachev, JHEP 0802, 062 (2008). arXiv:0708.3170 [hep-th]

31. O. Aharony, K. Peeters, J. Sonnenschein, M. Zamaklar, JHEP 0802, 071 (2008). arXiv:0709.3948 [hep-th]

32. N. Evans, K.-Y. Kim, M. Magou, Y. Seo, S.-J. Sin, JHEP 1209, 045 (2012). arXiv:1204.5640 [hep-th]

33. Y. Seo, J.P. Shock, S.-J. Sin, D. Zoakos, JHEP 1003, 115 (2010). arXiv:0912.4013 [hep-th]

34. D. Albrecht, J. Erlich, Phys. Rev. D 82, 095002 (2010). arXiv:1007.3431 [hep-ph]

35. K.-I. Kim, Y. Kim, S.H. Lee, J. Korean Phys. Soc. 55, 1381 (2009). arXiv:0709.1772 [hep-ph]

36. S.S. Afonin, A.A. Andrianov, D. Espriu, Phys. Lett. B 745, 52 (2015). arXiv:1503.08606 [hep-ph]

37. D. Albrecht, J. Erlich, R.J. Wilcox, Phys. Rev. D 85, 114012 (2012). arXiv: 1112.5643 [hep-ph]

38. H. Nishihara, M. Harada, Phys. Rev. D 89, 076001 (2014). arXiv: 1401.2928 [hep-ph]

39. H. Nishihara, M. Harada, Phys. Rev. D 90, 115027 (2014). arXiv: 1407.7344

40. C.P. Herzog, Phys. Rev. Lett. 98, 091601 (2007). arXiv:hep-th/0608151

41. C.A. Ballon Bayona, H. Boschi-Filho, N.R.F. Braga, L.A. Pando Zayas, Phys. Rev. D 77, 046002 (2008). arXiv:0705.1529 [hep-th]

42. B.-H. Lee, C. Park, S.-J. Sin, JHEP 0907, 087 (2009). arXiv:0905.2800 [hep-th]

43. K. Jo, B.-H. Lee, C. Park, S.-J. Sin, JHEP 1006, 022 (2010). arXiv:0909.3914 [hep-ph]

44. P. Colangelo, F. Giannuzzi, S. Nicotri, Phys. Rev. D 83, 035015 (2011). arXiv:1008.3116 [hep-ph]

45. C. Park, D.-Y. Gwak, B.-H. Lee, Y. Ko, S. Shin, Phys. Rev. D 84, 046007 (2011). arXiv:1104.4182 [hep-th]

46. Y. Kim, B.-H. Lee, S. Nam, C. Park, S.-J. Sin, Phys. Rev. D 76, 086003 (2007). arXiv:0706.2525 [hep-ph]

47. B.-H. Lee, Sh. Mamedov, S. Nam, C. Park, JHEP 1308, 045 (2013)

48. B.-H. Lee, Sh Mamedov, C. Park, Int. J. Mod. Phys. A 29, 1450170 (2014). arXiv:1402.6061 [hep-th]

49. B.-H. Lee, C. Park, S. Nam, JHEP 1505, 011 (2015). arXiv: 1412.3097 [hep-ph]

50. B.-H. Lee, C. Park, Phys. Lett. B 746, 202 (2015). arXiv:1503.03615 [hep-th]

51. Y. Kim, Y. Seo, I.J. Shin, S.-J. Sin, Holographic meson mass in asymmetric dense matter (2011). arXiv:1108.2751 [hep-ph]

52. N. Maru, M. Tachibana, Eur. Phys. J. C 63, 123 (2009). arXiv:0904.3816 [hep-ph]

53. C. Park, Phys. Lett. B 708, 324 (2012). arXiv:1112.0386 [hep-th]

54. H.R. Grigoryan, A.V. Radyushkin, Phys. Lett. B 650, 421 (2007). arXiv:hep-ph/0703069

55. A. Mammarella, M. Mannarelli, Phys. Rev. D 92, 085025 (2015). arxiv:1507.02934 [hep-ph]

56. B.-H. Lee, C. Park, S. Shin, JHEP 1012, 071 (2010). arXiv: 1010.1109 [hep-th]

57. C. Park, B.-H. Lee, S. Shin, Phys. Rev. D 85, 106005 (2012). arXiv:1112.2177 [hep-th]

58. H.R. Grigoryan, A.V. Radyushkin, Phys. Rev. D 76, 115007 (2007). arXiv:0709.0500 [hep-ph]

59. H.C. Kim, Y. Kim, U. Yakhshiev, JHEP 0911, 034 (2009). arXiv:0908.3406 [hep-ph] 